\title{
Prevention and Control of Seasonal Influenza with Vaccines: Recommendations of the Advisory Committee on Immunization Practices, United States, 2021-22 Influenza Season
}

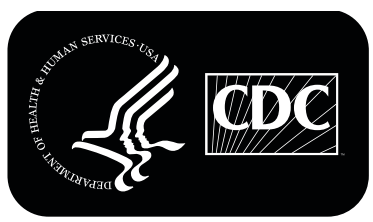




\section{CONTENTS}

Introduction . .2

Methods ....3

Primary Changes and Updates . .7

Recommendations for the Use of Influenza Vaccines, 2021-22...........8

Influenza Vaccine Composition and Available Vaccines ....................... 17

Storage and Handling of Influenza Vaccines. 20

Additional Sources of Information Regarding Influenza and Influenza Vaccines 21

References 22

\section{CDC Adoption of ACIP Recommendations for MMWR Recommendations and Reports, MMWR Policy Notes, and Immunization Schedules (Child/Adolescent, Adult)}

Recommendations for routine use of vaccines in children, adolescents, and adults are developed by the Advisory Committee on Immunization Practices (ACIP). ACIP is chartered as a Federal Advisory Committee to provide expert external advice and guidance to the Director of CDC on use of vaccines and related agents for the control of vaccine preventable diseases in the civilian population of the United States. Recommendations for routine use of vaccines in children and adolescents are harmonized to the greatest extent possible with recommendations made by the American Academy of Pediatrics (AAP), the American Academy of Family Physicians (AAFP), and the American College of Obstetricians and Gynecologists (ACOG). Recommendations for routine use of vaccinations in adults are harmonized with recommendations of AAFP, ACOG, and the American College of Physicians (ACP). ACIP recommendations approved by the CDC Director become agency guidelines on the date published in the Morbidity and Mortality Weekly Report (MMWR). Additional information is available at https://www.cdc.gov/vaccines/acip.

The MMWR series of publications is published by the Center for Surveillance, Epidemiology, and Laboratory Services, Centers for Disease Control and Prevention (CDC), U.S. Department of Health and Human Services, Atlanta, GA 30329-4027.

Suggested citation: [Author names; first three, then et al., if more than six.] [Title]. MMWR Recomm Rep 2021;70(No. RR-\#):[inclusive page numbers].

\author{
Centers for Disease Control and Prevention \\ Rochelle P. Walensky, MD, MPH, Director \\ Debra Houry, MD, MPH, Acting Principal Deputy Director \\ Daniel B. Jernigan, MD, MPH, Acting Deputy Director for Public Health Science and Surveillance \\ Rebecca Bunnell, PhD, MEd, Director, Office of Science \\ Jennifer Layden, MD, PhD, Deputy Director, Office of Science \\ Michael F. Iademarco, MD, MPH, Director, Center for Surveillance, Epidemiology, and Laboratory Services
}

MMWR Editorial and Production Staff (Serials)

\begin{abstract}
Charlotte K. Kent, PhD, MPH, Editor in Chief Christine G. Casey, MD, Editor

Mary Dott, MD, MPH, Online Editor

Terisa F. Rutledge, Managing Editor

David C. Johnson, Lead Technical Writer-Editor

Catherine B. Lansdowne, MS, Project Editor
\end{abstract}

Matthew L. Boulton, MD, MPH

Carolyn Brooks, ScD, MA

Jay C. Butler, MD

Virginia A. Caine, MD

Jonathan E. Fielding, MD, MPH, MBA

David W. Fleming, MD
Martha F. Boyd, Lead Visual Information Specialist Alexander J. Gottardy, Maureen A. Leahy,

Julia C. Martinroe, Stephen R. Spriggs, Tong Yang, Visual Information Specialists

Quang M. Doan, MBA, Phyllis H. King, Terraye M. Starr, Moua Yang, Information Technology Specialists

MMWR Editorial Board

Timothy F. Jones, MD, Chairman

William E. Halperin, MD, DrPH, MPH Jewel Mullen, MD, MPH, MPA Jeff Niederdeppe, $\mathrm{PhD}$ Celeste Philip, MD, MPH Patricia Quinlisk, MD, MPH

Patrick L. Remington, MD, MPH
Ian Branam, MA, Ginger Redmon, MA, Acting Lead Health Communication Specialists Shelton Bartley, MPH,

Lowery Johnson, Amanda Ray, Jacqueline N. Sanchez, MS,

Health Communication Specialists Will Yang, MA, Visual Information Specialist

Carlos Roig, MS, MA William Schaffner, MD Nathaniel Smith, MD, MPH Morgan Bobb Swanson, BS Abbigail Tumpey, MPH 


\title{
Prevention and Control of Seasonal Influenza with Vaccines: Recommendations of the Advisory Committee on Immunization Practices, United States, 2021-22 Influenza Season
}

\author{
Lisa A. Grohskopf, MD ${ }^{1}$, Elif Alyanak, $\mathrm{MPH}^{1,2}$, Jill M. Ferdinands, $\mathrm{PhD}^{1}$, Karen R. Broder, $\mathrm{MD}^{3}$, Lenee H. Blanton, $\mathrm{MPH}^{1}$,
} H. Keipp Talbot, $\mathrm{MD}^{4}$, Alicia M. Fry, $\mathrm{MD}^{1}$ ${ }^{1}$ Influenza Division, National Center for Immunization and Respiratory Diseases, CDC; ${ }^{2}$ Abt Associates, Atlanta, Georgia; ${ }^{3}$ Immunization Safety Office, National
Center for Emerging and Zoonotic Infectious Diseases, CDC; ${ }^{4}$ Division of Infectious Diseases, Vanderbilt University Medical Center, Nashville, Tennessee

\section{Summary}

This report updates the 2020-21 recommendations of the Advisory Committee on Immunization Practices (ACIP) regarding the use of seasonal influenza vaccines in the United States (MMWR Recomm Rep 2020;69[No. RR-8]). Routine annual influenza vaccination is recommended for all persons aged $\geq 6$ months who do not have contraindications. For each recipient, a licensed and age-appropriate vaccine should be used. ACIP makes no preferential recommendation for a specific vaccine when more than one licensed, recommended, and age-appropriate vaccine is available. During the 2021-22 influenza season, the following types of vaccines are expected to be available: inactivated influenza vaccines (IIV4s), recombinant influenza vaccine (RIV4), and live attenuated influenza vaccine (LAIV4).

The 2021-22 influenza season is expected to coincide with continued circulation of SARS-CoV-2, the virus that causes COVID-19. Influenza vaccination of persons aged $\geq 6$ months to reduce prevalence of illness caused by influenza will reduce symptoms that might be confused with those of COVID-19. Prevention of and reduction in the severity of influenza illness and reduction of outpatient visits, hospitalizations, and intensive care unit admissions through influenza vaccination also could alleviate stress on the U.S. health care system. Guidance for vaccine planning during the pandemic is available at https://www. cdc.gov/vaccines/pandemic-guidancelindex.html. Recommendations for the use of COVID-19 vaccines are available at https:// www.cdc.gov/vaccines/hcplacip-recs/vacc-specific/covid-19.html, and additional clinical guidance is available at https://www.cdc. gov/vaccines/covid-19/clinical-considerations/covid-19-vaccines-us.html.

Updates described in this report reflect discussions during public meetings of ACIP that were held on October 28, 2020; February 25, 2021; and June 24, 2021. Primary updates to this report include the following six items. First, all seasonal influenza vaccines available in the United States for the 2021-22 season are expected to be quadrivalent. Second, the composition of 2021-22 U.S. influenza vaccines includes updates to the influenza A(H1N1)pdm09 and influenza A(H3N2) components. U.S.-licensed influenza vaccines will contain hemagglutinin derived from an influenza A/Victoria/2570/2019 (H1N1)pdm09-like virus (for egg-based vaccines) or an influenza A/Wisconsin/588/2019 (H1N1)pdm09-like virus (for cell culture-based and recombinant vaccines), an influenza A/Cambodiale0826360/2020 (H3N2)-like virus, an influenza B/Washington/02/2019 (Victoria lineage)-like virus, and an influenza B/Phuket/3073/2013 (Yamagata lineage)-like virus. Third, the approved age indication for the cell culture-based inactivated influenza vaccine, Flucelvax Quadrivalent (ccIIV4), has been expanded from ages $\geq 4$ years to ages $\geq 2$ years. Fourth, discussion of administration of influenza vaccines with other vaccines includes considerations for coadministration of influenza vaccines and COVID-19 vaccines. Providers should also consult current ACIP COVID-19 vaccine recommendations and CDC guidance concerning coadministration of these vaccines with influenza vaccines. Vaccines that are given at the same time should be administered in separate anatomic sites. Fifth, guidance concerning timing of influenza vaccination now states that vaccination soon after vaccine becomes available can be considered for pregnant women in the third trimester. As previously recommended, children who need 2 doses (children aged 6 months through 8 years who have never received influenza vaccine or who have not previously received a lifetime total of $\geq 2$ doses) should receive their first dose as soon as possible after vaccine becomes available to allow the second dose (which must be administered $\geq 4$ weeks later) to be received by the end of October. For nonpregnant adults, vaccination in July and August should be avoided unless there is concern that later vaccination might not be possible. Sixth, contraindications and precautions to the use of ccIIV4 and RIV4 have been modified, specifically with regard to persons with a

Corresponding author: Lisa Grohskopf, Influenza Division, National Center for Immunization and Respiratory Diseases, CDC. Telephone: 404-639-2552; Email: lgrohskopf@cdc.gov. 
history of severe allergic reaction (e.g., anaphylaxis) to an influenza vaccine. A history of a severe allergic reaction to a previous dose of any egg-based IIV, LAIV, or RIV of any valency is a precaution to use of ccIIV4. A history of a severe allergic reaction to a previous dose of any egg-based IIV, ccIIV, or LAIV of any valency is a precaution to use of RIV4. Use of ccIIV4 and RIV4 in such instances should occur in an inpatient or outpatient medical setting under supervision of a provider who can recognize and manage a severe allergic reaction; providers can also consider consulting with an allergist to help identify the vaccine component responsible for the reaction. For ccIIV4, history of a severe allergic reaction (e.g., anaphylaxis) to any ccIIV of any valency or any component of ccIIV4 is a contraindication to future use of ccIIV4. For RIV4, history of a severe allergic reaction (e.g., anaphylaxis) to any RIV of any valency or any component of RIV4 is a contraindication to future use of RIV4.

This report focuses on recommendations for the use of vaccines for the prevention and control of seasonal influenza during the 2021-22 influenza season in the United States. A brief summary of the recommendations and a link to the most recent Background Document containing additional information are available at https://www.cdc.gov/vaccines/hcplacip-recs/vacc-specific/flu. html. These recommendations apply to U.S.-licensed influenza vaccines used according to Food and Drug Administration-licensed indications. Updates and other information are available from CDC's influenza website (https://www.cdc.gov/flu); vaccination and health care providers should check this site periodically for additional information.

\section{Introduction}

Influenza viruses typically circulate annually in the United States, most commonly from the late fall through the early spring. Most persons who become ill after influenza virus infection recover without serious complications or sequelae. However, influenza can be associated with serious illnesses, hospitalizations, and deaths, particularly among older adults, very young children, pregnant women, and persons of all ages with certain chronic medical conditions (1-7). Influenza also is an important cause of missed work and school $(8-10)$. Routine annual influenza vaccination for all persons aged $\geq 6$ months who do not have contraindications has been recommended by $\mathrm{CDC}$ and the Advisory Committee on Immunization Practices (ACIP) since 2010 (11).

Vaccination provides important protection from influenza illness and its potential complications. The effectiveness of influenza vaccination varies depending on several factors, such as the age and health of the recipient; the type of vaccine administered; the types, subtypes (for influenza $A$ ), and lineages (for influenza B) of circulating influenza viruses; and the degree of similarity between circulating viruses and those included in the vaccine (12). During the six influenza seasons from 2010-11 through 2015-16, influenza vaccination prevented an estimated 1.6-6.7 million illnesses, 790,000-3.1 million outpatient medical visits, 39,000-87,000 hospitalizations, and $3,000-10,000$ respiratory and circulatory deaths each season in the United States (13). During the severe 2017-18 season, notable for an unusually long duration of widespread high influenza activity throughout the United States and higher rates of outpatient visits and hospitalizations compared with recent seasons, vaccination prevented an estimated 7.1 million illnesses, 3.7 million medical visits, 109,000 hospitalizations, and 8,000 deaths (14), despite an overall estimated vaccine effectiveness of $38 \%$ (62\% against influenza $A[\mathrm{H} 1 \mathrm{~N} 1] \mathrm{pdm} 09$ viruses, $22 \%$ against influenza $\mathrm{A}[\mathrm{H} 3 \mathrm{~N} 2]$ viruses, and $50 \%$ against influenza B viruses) (14).

In late 2019, a novel coronavirus, SARS-CoV-2, emerged as a cause of severe respiratory illness (15). In March 2020, the World Health Organization (WHO) declared COVID-19, the illness caused by SARS-CoV-2, a global pandemic (16). As of August 12, 2021, approximately 36.3 million cases of COVID-19 had been reported in the United States, including approximately 617,000 deaths (17). Although influenza activity during the 2020-21 season was low throughout the United States (18), the timing and intensity of the upcoming 2021-22 influenza season cannot be predicted. Influenza vaccination remains an important tool for the prevention of potentially severe respiratory illness, which might decrease stress on the U.S. health care system during ongoing circulation of SARS-CoV-2. Guidance for vaccine planning during the COVID-19 pandemic is available at https://www.cdc.gov/ vaccines/pandemic-guidance/index.html.

This report updates the 2020-21 ACIP recommendations regarding the use of seasonal influenza vaccines (19) and provides recommendations and guidance for vaccine providers regarding the use of influenza vaccines in the United States for the 2021-22 season. Various formulations of influenza vaccines are available (Table 1). Contraindications and precautions for the use of influenza vaccines are summarized (Tables 2 and 3). Abbreviations are used in this report to denote the various types of vaccines (Box).

This report focuses on recommendations and guidance for the use of seasonal influenza vaccines for the prevention and control of influenza during the 2021-22 season in the United States. A summary of these recommendations and a Background Document containing additional information on influenza, influenza-associated illness, and influenza vaccines are available at https://www.cdc.gov/vaccines/hcp/acip-recs/ vacc-specific/flu.html. 


\begin{tabular}{|c|c|c|c|c|c|}
\hline Trade name (manufacturer) & Presentations & Age indication & $\begin{array}{l}\mu \mathrm{g} \mathrm{HA} \text { (IIV4s and RIV4) } \\
\text { or virus count (LAIV4) } \\
\text { for each vaccine virus } \\
\text { (per dose) }\end{array}$ & Route & $\begin{array}{c}\text { Mercury } \\
\text { (from thimerosal, } \\
\text { if present), } \\
\mu \mathrm{g} / 0.5 \mathrm{~mL}\end{array}$ \\
\hline \multicolumn{6}{|c|}{ IIV4 (standard-dose, egg-based vaccines ${ }^{\dagger}$ ) } \\
\hline $\begin{array}{l}\text { Afluria Quadrivalent } \\
\text { (Seqirus) }\end{array}$ & $\begin{array}{r}0.25-\mathrm{mL} \mathrm{PFS}^{\S} \\
0.5-\mathrm{mL} \mathrm{PFS}^{\S} \\
5.0-\mathrm{mL} \mathrm{MDV}^{\S}\end{array}$ & $\begin{array}{r}6 \text { through } 35 \text { mos }^{\S} \\
\geq 3 \mathrm{yrs}^{\S} \\
\geq 6 \operatorname{mos}^{\S} \text { (needle/syringe) } \\
18 \text { through } 64 \text { yrs (jet injector) }\end{array}$ & $\begin{array}{r}7.5 \mu \mathrm{g} / 0.25 \mathrm{~mL} \\
15 \mu \mathrm{g} / 0.5 \mathrm{~mL} \\
15 \mu \mathrm{g} / 0.5 \mathrm{~mL}\end{array}$ & $\begin{array}{l}I_{M} \text { थ } \\
I^{9} \\
I M^{\text {? }}\end{array}$ & $\frac{-}{24.5}$ \\
\hline $\begin{array}{l}\text { Fluarix Quadrivalent } \\
\text { (GlaxoSmithKline) }\end{array}$ & $0.5-\mathrm{mL}$ PFS & $\geq 6 \mathrm{mos}$ & $15 \mu \mathrm{g} / 0.5 \mathrm{~mL}$ & $\mathrm{IM}^{\text {9 }}$ & - \\
\hline $\begin{array}{l}\text { FluLaval Quadrivalent } \\
\text { (GlaxoSmithKline) }\end{array}$ & $0.5-\mathrm{mL}$ PFS & $\geq 6 \mathrm{mos}$ & $15 \mu \mathrm{g} / 0.5 \mathrm{~mL}$ & $I M M^{9}$ & - \\
\hline $\begin{array}{l}\text { Fluzone Quadrivalent } \\
\text { (Sanofi Pasteur) }\end{array}$ & $\begin{array}{r}0.5-\mathrm{mL} \mathrm{PFS}^{* *} \\
0.5-\mathrm{mL} \mathrm{SDV} * * \\
5.0-\mathrm{mL} \mathrm{MDV}^{* *}\end{array}$ & $\begin{array}{l}\geq 6 \operatorname{mos}^{* *} \\
\geq 6 \operatorname{mos}^{* *} \\
\geq 6 \operatorname{mos}^{* *}\end{array}$ & $\begin{array}{r}15 \mu \mathrm{g} / 0.5 \mathrm{~mL} \\
15 \mu \mathrm{g} / 0.5 \mathrm{~mL} \\
15 \mu \mathrm{g} / 0.5 \mathrm{~mL} \\
7.5 \mu \mathrm{g} / 0.25 \mathrm{~mL}\end{array}$ & $\begin{array}{l}I_{M}^{\text {श }} \\
I^{9} \\
I^{9}\end{array}$ & $\frac{-}{25}$ \\
\hline \multicolumn{6}{|c|}{ ccllV4 (standard-dose, cell culture-based vaccine) } \\
\hline $\begin{array}{l}\text { Flucelvax Quadrivalent } \\
\text { (Seqirus) }\end{array}$ & $\begin{array}{r}0.5-\mathrm{mL} \text { PFS } \\
5.0-\mathrm{mL} \text { MDV }\end{array}$ & $\begin{array}{l}\geq 2 \mathrm{yrs} \\
\geq 2 \mathrm{yrs}\end{array}$ & $\begin{array}{l}15 \mu \mathrm{g} / 0.5 \mathrm{~mL} \\
15 \mu \mathrm{g} / 0.5 \mathrm{~mL}\end{array}$ & $\begin{array}{l}I^{9} \\
I^{9}\end{array}$ & $\overline{25}$ \\
\hline $\begin{array}{l}\text { HD-IIV4 (high-dose, egg-based v } \\
\text { Fluzone High-Dose Quadrivalent } \\
\text { (Sanofi Pasteur) }\end{array}$ & 0.7-mL PFS & $\geq 65$ yrs & $60 \mu \mathrm{g} / 0.7 \mathrm{~mL}$ & $\mathrm{IM}^{\text {9 }}$ & - \\
\hline $\begin{array}{l}\text { allV4 (standard-dose, egg-based } \\
\text { Fluad Quadrivalent } \\
\text { (Seqirus) }\end{array}$ & $\begin{array}{l}\text { with MF59 adjuvant } \\
0.5-\mathrm{mL} \text { PFS }\end{array}$ & $\geq 65$ yrs & $15 \mu \mathrm{g} / 0.5 \mathrm{~mL}$ & $\mathrm{IM}^{\text {थ }}$ & - \\
\hline $\begin{array}{l}\text { RIV4 (recombinant HA vaccine) } \\
\text { Flublok Quadrivalent } \\
\text { (Sanofi Pasteur) }\end{array}$ & $0.5-\mathrm{mL}$ PFS & $\geq 18 \mathrm{yrs}$ & $45 \mu \mathrm{g} / 0.5 \mathrm{~mL}$ & $I M M^{9}$ & - \\
\hline $\begin{array}{l}\text { LAIV4 (egg-based vaccine }{ }^{\dagger} \text { ) } \\
\text { FluMist Quadrivalent } \\
\text { (AstraZeneca) }\end{array}$ & $\begin{array}{r}0.2-\mathrm{mL} \text { prefilled } \\
\text { single-use } \\
\text { intranasal sprayer }\end{array}$ & 2 through 49 yrs & $\begin{array}{c}10^{6.5-7.5} \text { fluorescent focus } \\
\text { units } / 0.2 \mathrm{~mL}\end{array}$ & NAS & - \\
\hline
\end{tabular}

Abbreviations: ACIP = Advisory Committee on Immunization Practices; FDA = Food and Drug Administration; HA = hemagglutinin; IIV4 = inactivated influenza vaccine, quadrivalent; IM = intramuscular; LAIV4 = live attenuated influenza vaccine, quadrivalent; $M D V=$ multidose vial; NAS = intranasal; PFS = prefilled syringe; RIV4 = recombinant influenza vaccine, quadrivalent; SDV = single-dose vial.

* Vaccination providers should consult FDA-approved prescribing information for 2021-22 influenza vaccines for the most complete and updated information, including (but not limited to) indications, contraindications, warnings, and precautions. Package inserts for U.S.licensed vaccines are available at https://www.fda. gov/vaccines-blood-biologics/approved-products/vaccines-licensed-use-united-states. Availability and characteristics of specific products and presentations might change or differ from what is described in this table and in the text of this report.

† Although a history of severe allergic reaction (e.g., anaphylaxis) to egg is a labeled contraindication to the use of egg-based IIV4s and LAIV4, ACIP recommends that persons with a history of egg allergy may receive any licensed, recommended influenza vaccine that is otherwise appropriate for their age and health status. Those who report having had reactions to egg involving symptoms other than urticaria (e.g., angioedema or swelling, respiratory distress, lightheadedness, or recurrent emesis) or who required epinephrine or another emergency medical intervention should be vaccinated in an inpatient or outpatient medical setting (including but not necessarily limited to hospitals, clinics, health departments, and physician offices) supervised by a health care provider who is able to recognize and manage severe allergic reactions, if a vaccine other than ccllV4 or RIV4 is used.

$\S$ The dose volume for Afluria Quadrivalent is $0.25 \mathrm{~mL}$ for children aged 6 through 35 months and $0.5 \mathrm{~mL}$ for persons aged $\geq 3$ years.

I IM-administered influenza vaccines should be given by needle and syringe only, with the exception of the MDV presentation of Afluria Quadrivalent, which may alternatively be given by the PharmaJet Stratis jet injector for persons aged 18 through 64 years only. For adults and older children, the recommended site for IM influenza vaccination is the deltoid muscle. The preferred site for infants and young children is the anterolateral aspect of the thigh. Additional specific guidance regarding site selection and needle length for IM administration is available in the ACIP General Best Practice Guidelines for Immunization, available at https:// www.cdc.gov/vaccines/hcp/acip-recs/general-recs/index.html.

** Fluzone Quadrivalent is currently approved for ages 6 through 35 months at either $0.25 \mathrm{~mL}$ or $0.5 \mathrm{~mL}$ per dose; however, 0.25 -mL prefilled syringes are not expected to be available for the 2021-22 influenza season. If a prefilled syringe of Fluzone Quadrivalent is used for a child in this age group, the dose volume will be $0.5 \mathrm{~mL}$ per dose.

\section{Methods}

ACIP provides annual recommendations for the use of influenza vaccines for the prevention and control of influenza in the United States. The ACIP Influenza Work Group meets by teleconference once to twice per month throughout the year. Work group membership includes several voting members of ACIP, representatives of ACIP liaison organizations, and consultants. Discussions include topics such as influenza surveillance, vaccine effectiveness and safety, vaccination coverage, program feasibility, cost-effectiveness, and vaccine supply. Presentations are requested from invited experts, and published and unpublished data are discussed.

The Background Document that supplements this report is updated periodically to reflect recent additions to the literature 
TABLE 2. Contraindications and precautions to the use of influenza vaccines - United States, 2021-22 influenza season*

\section{Vaccine type}

Egg-based IIV4s

ccllV4

LAIV4
Contraindications

- History of severe allergic reaction (e.g., anaphylaxis) to any component of the vaccine ${ }^{\dagger}$ or to a previous dose of any influenza vaccine (i.e., any egg-based IIV, ccIIV, RIV, or LAIV) ${ }^{\S}$

- History of severe allergic reaction (e.g., anaphylaxis) to a previous dose of any ccllV or any component of ccllV4 ${ }^{\S}$

- History of severe allergic reaction (e.g., anaphylaxis) to a previous dose of any RIV or any component of RIV4§

\section{Precautions}

- Moderate or severe acute illness with or without fever

- History of Guillain-Barré syndrome within 6 weeks of receipt of influenza vaccine

- Moderate or severe acute illness with or without fever

- History of Guillain-Barré syndrome within 6 weeks of receipt of influenza vaccine

- History of severe allergic reaction to a previous dose of any other influenza vaccine (i.e., any egg-based IIV, RIV, or LAIV)?

- Moderate or severe acute illness with or without fever

- History of Guillain-Barré syndrome within 6 weeks of receipt of influenza vaccine

- History of severe allergic reaction to a previous dose of any other influenza vaccine (i.e., any egg-based IIV, ccIIV, or LAIV)

- Moderate or severe acute illness with or without fever

- History of severe allergic reaction (e.g., anaphylaxis) to any component of the vaccine ${ }^{\dagger}$ or to a previous dose of any influenza vaccine (i.e., any egg-based IIV, ccIIV, RIV, or LAIV) ${ }^{\S}$

- Concomitant aspirin or salicylate-containing therapy in children and adolescents $\S$

- Children aged 2 through 4 years who have received a diagnosis of asthma or whose parents or caregivers report that a health care provider has told them during the preceding 12 months that their child had wheezing or asthma or whose medical record indicates a wheezing episode has occurred during the preceding 12 months

- Children and adults who are immunocompromised due to any cause, including but not limited to immunosuppression caused by medications, congenital or acquired immunodeficiency states, HIV infection, anatomic asplenia, or functional asplenia (e.g., due to sickle-cell anemia)

- Close contacts and caregivers of severely immunosuppressed persons who require a protected environment

- Pregnancy

- Persons with active communication between the CSF and the oropharynx, nasopharynx, nose, or ear or any other cranial CSF leak

- Persons with cochlear implants**

- Receipt of influenza antiviral medication within the previous 48 hours for oseltamivir and zanamivir, previous 5 days for peramivir, and previous 17 days for baloxavirt†

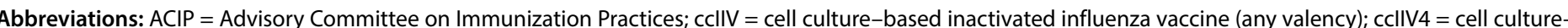

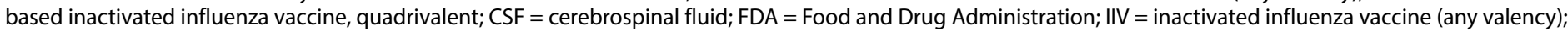

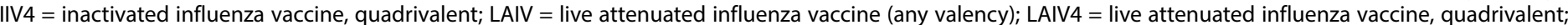
RIV = recombinant influenza vaccine (any valency); RIV4 = recombinant influenza vaccine, quadrivalent.

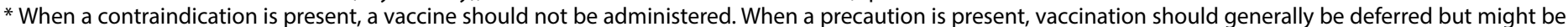

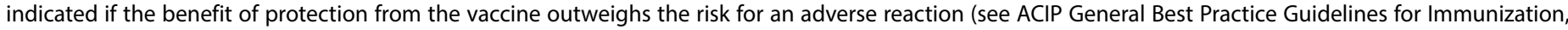

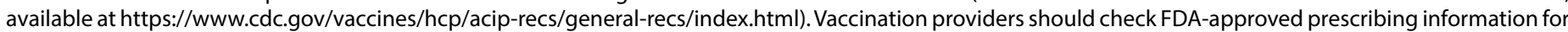

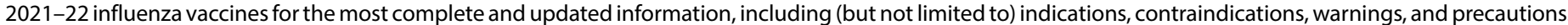

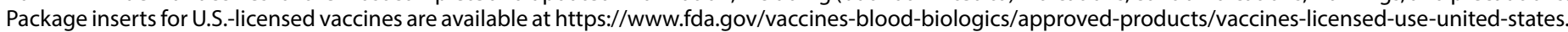

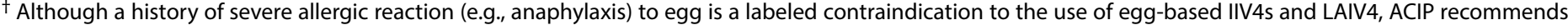

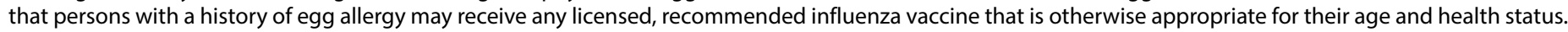

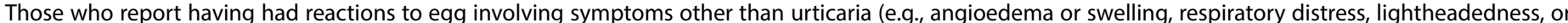

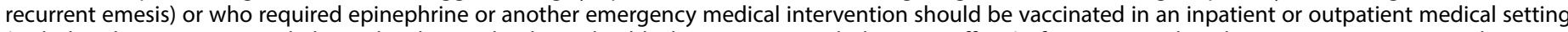

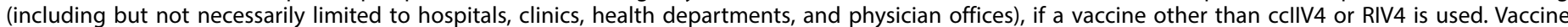
administration should be supervised by a health care provider who is able to recognize and manage severe allergic reactions.

$\S$ Labeled contraindication noted in package insert.

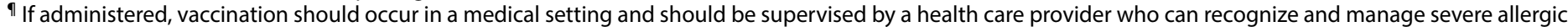
reactions. Providers can consider consultation with an allergist in such cases, to assist in identification of the component responsible for the allergic reaction.

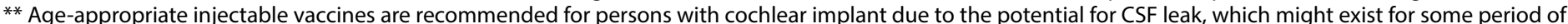

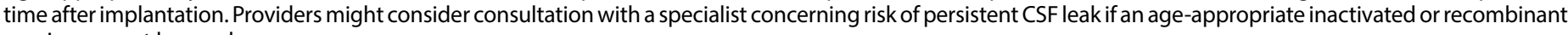
vaccine cannot be used.

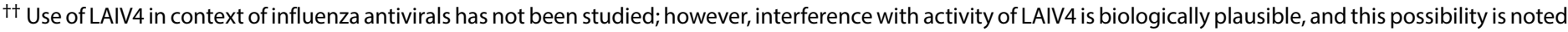

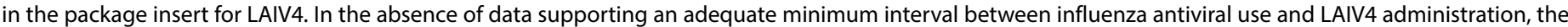

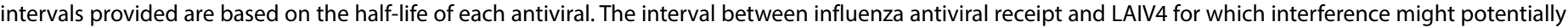

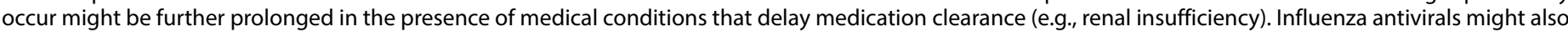

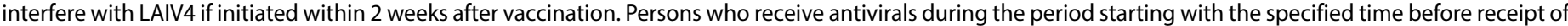
LAIV4 through 2 weeks after receipt of LAIV4 should be revaccinated with an age-appropriate IIV or RIV4. 
TABLE 3. Influenza vaccine contraindications and precautions for persons with a history of severe allergic reaction to a previous dose of an influenza vaccine* - United States, 2021-22 influenza season

Vaccine (of any valency) associated with previous severe allergic reaction (e.g., anaphylaxis)

Any egg-based IIV or LAIV

Any ccllV

Any RIV

Unknown influenza vaccine

Available 2021-22 influenza vaccines

Egg-based IIV4s and LAIV4

Contraindication $^{\dagger}$

Contraindication $^{\dagger}$

Contraindication $^{\dagger}$
ccllV4

Precaution $\$$

Contraindication $^{\dagger}$

Precaution $^{\S}$

Allergist consultation recommended

\section{RIV4}

Precaution $^{\S}$

Precaution $\$$

Contraindication $^{\dagger}$

Abbreviations: ACIP = Advisory Committee on Immunization Practices; ccIIV = cell culture-based inactivated influenza vaccine (any valency); ccIIV4 = cell culturebased inactivated influenza vaccine, quadrivalent; FDA = Food and Drug Administration; IIV = inactivated influenza vaccine (any valency); IIV4 = inactivated influenza vaccine, quadrivalent; LAIV = live attenuated influenza vaccine (any valency); LAIV4 = live attenuated influenza vaccine, quadrivalent; RIV = recombinant influenza vaccine (any valency); RIV4 = recombinant influenza vaccine, quadrivalent.

* Vaccination providers should check FDA-approved prescribing information for 2021-22 influenza vaccines for the most complete and updated information, including (but not limited to) indications, contraindications, warnings, and precautions. Package inserts for U.S.-licensed vaccines are available at https://www.fda.gov/ vaccines-blood-biologics/approved-products/vaccines-licensed-use-united-states.

+ When a contraindication is present, a vaccine should not be administered, consistent with ACIP General Best Practice Guidelines for Immunization (Kroger A, Bahta L, Hunter P. General best practice guidelines for immunization: best practices guidance of the Advisory Committee on Immunization Practices [ACIP]. https://www. cdc.gov/vaccines/hcp/acip-recs/general-recs/index.html ). In addition to the contraindications based on history of severe allergic reaction to influenza vaccines that are noted in the Table, each individual influenza vaccine is contraindicated for persons who have had a severe allergic reaction (e.g., anaphylaxis) to any component of that vaccine. Vaccine components can be found in package inserts. Although a history of severe allergic reaction (e.g., anaphylaxis) to egg is a labeled contraindication to the use of egg-based IIV4s and LAIV4, ACIP recommends that persons with a history of egg allergy may receive any licensed, recommended influenza vaccine that is otherwise appropriate for their age and health status. Those who report having had reactions to egg involving symptoms other than urticaria (e.g., angioedema or swelling, respiratory distress, lightheadedness, or recurrent emesis) or who required epinephrine or another emergency medical intervention should be vaccinated in an inpatient or outpatient medical setting (including but not necessarily limited to hospitals, clinics, health departments, and physician offices), if a vaccine other than ccllV4 or RIV4 is used. Vaccine administration should be supervised by a health care provider who is able to recognize and manage severe allergic reactions.

$\S$ When a precaution is present, vaccination should generally be deferred but might be indicated if the benefit of protection from the vaccine outweighs the risk for an adverse reaction, consistent with ACIP General Best Practice Guidelines for Immunization (Kroger A, Bahta L, Hunter P. General best practice guidelines for immunization: best practices guidance of the Advisory Committee on Immunization Practices [ACIP]. https://www.cdc.gov/vaccines/hcp/acip-recs/general-recs/ index.html). Providers can consider using the following vaccines in these instances; however, vaccination should occur in an inpatient or outpatient medical setting with supervision by a health care provider who is able to recognize and manage severe allergic reactions: 1) for persons with a history of severe allergic reaction (e.g., anaphylaxis) to any egg-based IIV or LAIV of any valency, the provider can consider administering ccllV4 or RIV4; 2 ) for persons with a history of severe allergic reaction (e.g., anaphylaxis) to any cclIV of any valency, the provider can consider administering RIV4; and 3) for persons with a history of severe allergic reaction (e.g., anaphylaxis) to any RIV of any valency, the provider can consider administering ccllV4. Providers can also consider consulting with an allergist to help determine which vaccine component is responsible for the allergic reaction.

related to recommendations made in previous seasons and minor changes in guidance for the use of influenza vaccines (e.g., guidance for timing of vaccination and other programmatic issues, guidance for dosage in specific populations, guidance for selection of vaccines for specific populations that are already recommended for vaccination, and changes that reflect use that is consistent with indications and prescribing information licensed by the Food and Drug Administration [FDA]). The summary included in the Background Document for such topics is not a systematic review; it is intended to provide an overview of current literature, with updated articles being identified primarily through a broad search for Englishlanguage articles on influenza and influenza vaccines. In general, systematic review and evaluation of evidence using the Grading of Recommendations Assessment, Development and Evaluation (GRADE) approach (20) is performed for new recommendations or substantial changes in the current recommendations (e.g., expansion of the recommendation for influenza vaccination to new populations not previously recommended for vaccination or potential preferential recommendations for specific vaccines).

Primary updates and changes to the recommendations described in this report include 1) discussion of influenza vaccines expected to be available for the 2021-22 influenza season; 2) the vaccine virus composition for 2021-22 U.S. seasonal influenza vaccines; 3) recent regulatory actions, including one influenza vaccine labeling change that occurred after the publication of the 2020-21 ACIP influenza statement (19); 4) considerations for influenza vaccination during the COVID-19 pandemic; 5) updates to the recommendations concerning timing of vaccination; and 6) updates to the discussion of contraindications and precautions to influenza vaccines. Information relevant to these changes includes the following:

1. Availability of specific types and brands of licensed seasonal influenza vaccines in the United States is determined by the manufacturers of the vaccines. Information presented concerning vaccines expected to be available and their approved indications and usage reflects current knowledge and is subject to change.

2. Recommendations for the composition of Northern Hemisphere influenza vaccines are made by $\mathrm{WHO}$, which organizes a consultation, generally in February of each year. Surveillance data are reviewed, and candidate vaccine viruses are discussed. Information concerning the WHO meeting of February 26, 2021, for selection of the 2021-22 Northern Hemisphere vaccine viruses is 
- Main influenza vaccine types include:

$\circ$ IIV = inactivated influenza vaccine

- RIV = recombinant influenza vaccine

- LAIV = live attenuated influenza vaccine

- Numerals following letter abbreviations indicate valency (the number of influenza virus hemagglutinin [HA] antigens represented in the vaccine):

$\circ 4$ for quadrivalent vaccines: one $\mathrm{A}(\mathrm{H} 1 \mathrm{~N} 1)$, one $\mathrm{A}(\mathrm{H} 3 \mathrm{~N} 2)$, and two $\mathrm{B}$ viruses (one from each lineage)

- 3 for trivalent vaccines: one $\mathrm{A}(\mathrm{H} 1 \mathrm{~N} 1)$, one $\mathrm{A}(\mathrm{H} 3 \mathrm{~N} 2)$, and one $\mathrm{B}$ virus (from one lineage)

- All influenza vaccines expected to be available in the United States for the 2021-22 season are quadrivalent vaccines. However, abbreviations for trivalent vaccines (e.g., IIV3) might be used in this document when discussing information specific to trivalent vaccines.

- Abbreviations for general vaccine categories (e.g., IIV) might be used when discussing information that is not specific to either trivalent or quadrivalent vaccines.

- Prefixes are used when necessary to refer to some specific IIVs:

$\circ$ a for adjuvanted inactivated influenza vaccine (e.g., aIIV3 and aIIV4)

$\circ$ cc for cell culture-based inactivated influenza vaccine (e.g., ccIIV3 and ccIIV4)

$\circ \mathbf{H D}$ for high-dose inactivated influenza vaccine (e.g., HD-IIV3 and HD-IIV4)

- SD for standard-dose inactivated influenza vaccine (e.g. SD-IIV3 and SD-IIV4)

available at https:/www.who.int/publications/m/item/ recommended-composition-of-influenza-virus-vaccinesfor-use-in-the-2021-2022-northern-hemisphere-influenzaseason. Subsequently, FDA, which has regulatory authority over vaccines in the United States, convenes a meeting of its Vaccines and Related Biological Products Advisory Committee (VRBPAC). This committee considers the recommendations of WHO, reviews and discusses similar data, and makes a final decision regarding vaccine virus composition of influenza vaccines licensed and marketed in the United States. Materials from the VRBPAC discussion of March 5, 2021, during which the composition of the 2021-22 U.S. influenza vaccines was discussed, are available at https://www.fda.gov/advisory-committees/ advisory-committee-calendar/vaccines-and-relatedbiological-products-advisory-committee-march-5-2021meeting-announcement\#event-information.

3. Regarding recommendations concerning newly licensed influenza vaccines and changes to the licensed indications for existing vaccines, ACIP relies on FDA for review of safety, immunogenicity, and efficacy and effectiveness data pertaining to licensure and labeling of influenza vaccines. Regulatory information pertinent to the change in age indication for Flucelvax Quadrivalent discussed in this report is available at https://www.fda.gov/ vaccines-blood-biologics/vaccines/flucelvax-quadrivalent.

4. Guidance concerning timing of influenza vaccination relative to administration of COVID-19 vaccines is coordinated with current ACIP recommendations and
CDC guidance for the use of COVID-19 vaccines. This information might change as data and clinical experience with COVID-19 vaccines evolve. ACIP recommendations for the use of COVID-19 vaccines are available at https:/www.cdc.gov/vaccines/hcp/acip-recs/ vacc-specific/covid-19.html. Interim clinical guidance for the use of COVID-19 vaccines is available at https:// www.cdc.gov/vaccines/covid-19/clinical-considerations/ covid-19-vaccines-us.html. These pages should be checked periodically for updated information.

5. Guidance for the timing of influenza vaccination in specific populations is based on the potential timing of availability of influenza vaccines each season, typical timing of the U.S. influenza season, need for receipt of 2 doses separated by $\geq 4$ weeks before the start of the influenza season for some children aged 6 months through 8 years (those who have never received influenza vaccine or who have not previously received a lifetime total of $\geq 2$ doses), and lack of influenza vaccines for children aged $<6$ months (for whom maternal vaccination offers protection against influenza).

6. Information regarding indications, contraindications, and precautions for influenza vaccines is reviewed. Differences between ACIP recommendations and package labeling are noted in the text. Labeled contraindications and precautions are indicated (Table 2); contraindications and precautions recommended by ACIP also are noted. Package inserts for U.S.-licensed influenza vaccines are available at https://www.fda.gov/vaccines-blood-biologics/ vaccines/vaccines-licensed-use-united-states. 


\section{Primary Changes and Updates}

Routine annual influenza vaccination of all persons aged $\geq 6$ months who do not have contraindications continues to be recommended. ACIP makes no preferential recommendation for a specific influenza vaccine when more than one licensed, recommended, and age-appropriate vaccine is available. Updated information in this report includes the following:

1. All seasonal influenza vaccines expected to be available for the 2021-22 season are quadrivalent, containing hemagglutinin (HA) derived from one influenza $\mathrm{A}(\mathrm{H} 1 \mathrm{~N} 1) \mathrm{pdm} 09$ virus, one influenza $\mathrm{A}(\mathrm{H} 3 \mathrm{~N} 2)$ virus, one influenza $\mathrm{B} /$ Victoria lineage virus, and one influenza $B /$ Yamagata lineage virus.

2. The composition of the 2021-22 U.S. seasonal influenza vaccines includes updates to the influenza $\mathrm{A}(\mathrm{H} 1 \mathrm{~N} 1) \mathrm{pdm} 09$ and influenza A(H3N2) components. For the 2021-22 season, U.S.-licensed influenza vaccines will contain an influenza A/Victoria/2570/2019 (H1N1)pdm09like virus (for egg-based vaccines) or an influenza $\mathrm{A} / \mathrm{Wisconsin} / 588 / 2019$ (H1N1)pdm09-like virus (for cell culture-based and recombinant vaccines); an influenza A/Cambodia/e0826360/2020 (H3N2)like virus; an influenza B/Washington/02/2019 (Victoria lineage)-like virus; and an influenza B/Phuket/3073/2013 (Yamagata lineage)-like virus.

3. One labeling change is described. In March 2021, FDA granted approval for the use of Flucelvax Quadrivalent (cell culture-based quadrivalent inactivated influenza vaccine [ccIIV4]) for children aged 2 through $<4$ years. Flucelvax Quadrivalent had previously been approved for persons aged $\geq 4$ years; approval for those aged 4 through $<18$ years was based on immunogenicity data and required a postmarketing efficacy study. The new approval is based on a randomized observer-blinded clinical efficacy study conducted among children aged 2 through $<18$ years over three seasons, in which Flucelvax Quadrivalent demonstrated efficacy against laboratory-confirmed influenza of $54.6 \%$ (95\% confidence interval $[\mathrm{CI}]=45.7 \%-62.1 \%)$ compared with a noninfluenza control vaccine. Flucelvax Quadrivalent is now approved for persons aged $\geq 2$ years $(21)$.

4. Guidance regarding administration of influenza vaccines with other vaccines has been updated to reflect consideration for COVID-19 vaccination, which is expected to continue in the United States before and during the 2021-22 influenza season. Current guidance for the use of COVID-19 vaccines indicates that these vaccines can be coadministered with other vaccines, including influenza vaccines. Providers should consult current COVID-19 vaccine recommendations and guidance for up-todate information. ACIP recommendations for the use of COVID-19 vaccines are available at https:// www.cdc.gov/vaccines/hcp/acip-recs/vacc-specific/ covid-19.html. Interim clinical guidance for the use of COVID-19 vaccines is available at https://www.cdc. gov/vaccines/covid-19/clinical-considerations/covid19-vaccines-us.html. These pages should be checked periodically for updated information.

5. Guidance concerning timing of vaccination has been modified. For women in the third trimester of pregnancy, vaccination soon after vaccine becomes available can now be considered. As in previous seasons, children who need 2 doses of influenza vaccine administered $\geq 4$ weeks apart (those aged 6 months through 8 years who have never received influenza vaccine or who have not previously received a lifetime total of $\geq 2$ doses) are recommended to receive the first dose as soon as possible after vaccine becomes available. For nonpregnant adults, early vaccination (i.e., in July and August) should be avoided unless there is concern that later vaccination might not be possible.

6. Contraindications and precautions to the use of ccIIV4 and RIV4 have been modified, specifically with regard to persons with a history of severe allergic reaction (e.g., anaphylaxis) to an influenza vaccine. A history of a severe allergic reaction (e.g., anaphylaxis) to a previous dose of any egg-based IIV, LAIV, or RIV of any valency is a precaution to use of ccIIV4. A history of a severe allergic reaction (e.g., anaphylaxis) to a previous dose of any egg-based IIV, ccIIV, or LAIV of any valency is a precaution to use of RIV4. Use of ccIIV4 and RIV4 in such instances should occur in an inpatient or outpatient medical setting under supervision of a provider who can recognize and manage a severe allergic reaction; providers can also consider consulting with an allergist to help identify the vaccine component responsible for the reaction. For ccIIV4, history of a severe allergic reaction (e.g., anaphylaxis) to any ccIIV of any valency or any of component of ccIIV4 is a contraindication to future use of ccIIV4. For RIV4, history of a severe allergic reaction (e.g., anaphylaxis) to any RIV of any valency or any component of RIV4 is a contraindication to future use of RIV4. 


\section{Recommendations for the Use of Influenza Vaccines, 2021-22}

\section{Groups Recommended for Vaccination}

Routine annual influenza vaccination is recommended for all persons aged $\geq 6$ months who do not have contraindications. Recommendations regarding timing of vaccination, considerations for specific populations, the use of specific vaccines, and contraindications and precautions are summarized in the sections that follow.

\section{Timing of Vaccination}

Balancing considerations regarding the unpredictability of timing of onset of the influenza season and concerns that vaccine-induced immunity might wane over the course of a season (22-35), particularly for older adults $(22,24,27,31,32,35)$, vaccination is recommended to be offered by the end of October. Children aged 6 months through 8 years who require 2 doses (i.e., children in this age group who have never received influenza vaccine or who have not previously received a lifetime total of $\geq 2$ doses; see Children Aged 6 Months Through 8 Years) should receive their first dose as soon as possible after the vaccine becomes available to allow the second dose (which must be administered $\geq 4$ weeks later) to be received, ideally, by the end of October. Children of any age who require only 1 dose for the season should also ideally be vaccinated by the end of October; vaccination of these children may occur as soon as vaccine is available because there is less evidence to suggest that early vaccination is associated with waning immunity among children compared with adults $(24,25,28,33)$. Vaccination soon after vaccine becomes available may also be considered for pregnant women during the third trimester because vaccination of pregnant women reduces risk for influenza illness in their infants during the first months of life (a period during which they are too young to receive influenza vaccine) (36-40). For nonpregnant adults, influenza vaccination during July and August should be avoided unless there is concern that later vaccination might not be possible. Early vaccination might be associated with decreased vaccine effectiveness before the end of the influenza season, particularly among older adults $(22,24,27,32,35)$. Community vaccination programs should balance maximizing the likelihood of persistence of vaccine-induced protection through the season with avoiding missed opportunities to vaccinate or vaccinating after onset of influenza circulation occurs. Efforts should be structured to optimize vaccination coverage before influenza activity in the community begins. Vaccination should continue to be offered as long as influenza viruses are circulating and unexpired vaccine is available. To avoid missed opportunities for vaccination, providers should offer vaccination during routine health care visits and hospitalizations. No recommendation is made for revaccination (i.e., providing a booster dose) later in the season of persons who have already been fully vaccinated for the season, regardless of when the current season vaccine was received.

During the 2021-22 influenza season, it is expected that SARS-CoV-2 will continue to circulate in the United States, and COVID-19 vaccinations are expected to continue. Current guidance for the administration of COVID-19 vaccines (available at https://www.cdc.gov/vaccines/covid-19/clinicalconsiderations/covid-19-vaccines-us.html) indicates that these vaccines can be administered with other vaccines, including influenza vaccines; providers should consult this page for updated information. Guidance for vaccine planning during the pandemic is available at https:/www.cdc.gov/vaccines/ pandemic-guidance/index.html. Additional discussion of coadministration of influenza and COVID-19 vaccines can be found in the section on Administration of Influenza Vaccines with Other Vaccines.

Optimally, vaccination should occur before onset of influenza activity in the community. However, because timing of the onset, peak, and decline of influenza activity varies, the ideal time to start vaccinating cannot be predicted each season. Moreover, more than one outbreak might occur in a given community in a single year. In the United States, localized outbreaks that indicate the start of seasonal influenza activity can occur as early as October. However, in 27 (75\%) of 36 influenza seasons from 1982-83 through 2017-18, peak influenza activity (which often is close to the midpoint of influenza activity for the season) has not occurred until January or later, and in $21(58 \%)$ seasons, the peak was in February or later (41). Activity peaked in February in 15 (42\%) of these seasons $(41)$.

An increasing number of observational studies (22-35) have reported decreases in vaccine effectiveness with increasing time postvaccination within a single influenza season. Waning effects have not been observed consistently across age groups, influenza viruses (types, subtypes, and lineages), and seasons. Some studies suggest waning occurs to a greater degree with influenza $\mathrm{A}(\mathrm{H} 3 \mathrm{~N} 2)$ viruses than with influenza $\mathrm{A}(\mathrm{H} 1 \mathrm{~N} 1)$ or influenza $\mathrm{B}$ viruses $(27,29)$. This effect also might vary with recipient age; in some studies, waning was more pronounced among older adults $(22,24,27,32,35)$ and younger children (24). Relatively fewer reports include results specific to children $(24,25,28,33)$; findings suggestive of waning have been reported in some $(24,25,28)$ but not others (33). Rates of decline in vaccine effectiveness also varied. A multiseason (2011-12 through 2014-15) analysis from the 
U.S. Influenza Vaccine Effectiveness (U.S. Flu VE) Network found that vaccine effectiveness decreased by approximately $7 \%$ per month for influenza $\mathrm{A}(\mathrm{H} 3 \mathrm{~N} 2)$ and influenza $\mathrm{B}$ and $6 \%-11 \%$ per month for influenza $A(\mathrm{H} 1 \mathrm{~N} 1)$ pdm09 (26). Vaccine effectiveness remained greater than zero for at least 5-6 months after vaccination. In the Hospitalized Adult Influenza Vaccine Effectiveness Network (HAIVEN) during the 2015-16 through 2018-19 seasons, vaccine effectiveness declined by approximately $8 \%-9 \%$ per month for all adults and approximately $10 \%-11 \%$ per month for those aged $\geq 65$ years (35). An analysis of the 2010-11 through 2013-14 seasons noted estimated effectiveness ranging from $54 \%$ to $67 \%$ during days $0-180$ postvaccination; estimated vaccine effectiveness was not statistically significant during the period between days 181 and 365 (25). A third multiseason analysis (2010-11 through 2014-15) conducted in Europe noted a decline in vaccine effectiveness to $0 \%$ at 111 days postvaccination for influenza $A(H 3 N 2)$ viruses. Vaccine effectiveness against influenza $B$ viruses decreased more slowly, and vaccine effectiveness against influenza $\mathrm{A}(\mathrm{H} 1 \mathrm{~N} 1) \mathrm{pdm} 09$ viruses remained roughly stable at $50 \%-55 \%$ through the influenza season (29). A meta-analysis of 14 studies examining waning of influenza vaccine effectiveness using the test-negative design found a significant decline in effectiveness within 180 days following vaccination for influenza A (H3N2) and influenza $B$ but not for influenza $A(\mathrm{H} 1 \mathrm{~N} 1)$ (42). In addition to the factors observed to be associated with waning immunity across studies, observed decreases in protection might be at least in part attributable to bias, unmeasured confounding, or the late-season emergence of antigenic drift variants that are less well-matched to the vaccine viruses.

Variable data concerning the presence and rate of waning immunity after influenza vaccination, coupled with the unpredictable timing of the influenza season each year, prevent determination of an optimal time to vaccinate. Programmatic issues are also a consideration: although delaying vaccination might result in greater immunity later in the season, deferral also might result in missed opportunities to vaccinate as well as difficulties in vaccinating a population within a more constrained period. The potential contributions of these factors among persons aged $\geq 65$ years have been assessed using a simulated mathematical model examining various scenarios of vaccination timing, timing of onset of the influenza season, rate of waning, and vaccine effectiveness (43). In this model, during an influenza season beginning in October and peaking in January, delaying vaccination until October resulted in more hospitalizations if $>14 \%$ of persons aged $\geq 65$ years who would have been vaccinated in August or September failed to get vaccinated. However, these predictions varied considerably with assumed timing of season onset, rate of waning immunity, and vaccine effectiveness.
Vaccination efforts should continue throughout the season because the duration of the influenza season varies, and influenza activity might not occur in certain communities until February or March. Providers should offer influenza vaccine routinely, and organized vaccination campaigns should continue throughout the influenza season, including after influenza activity has begun in the community. Although vaccination by the end of October is recommended, vaccine administered in December or later, even if influenza activity has already begun, might be beneficial in most influenza seasons. Providers should still offer influenza vaccination to unvaccinated persons who have already become ill with influenza during the season because the vaccine might protect them against other circulating influenza viruses.

\section{Guidance for Use in Specific Populations and Situations}

\section{Populations at Higher Risk for Medical Complications Attributable to Severe Influenza}

All persons aged $\geq 6$ months who do not have contraindications should be vaccinated annually. However, vaccination to prevent influenza is particularly important for persons who are at increased risk for severe illness and complications from influenza and for influenza-related outpatient, emergency department, or hospital visits. When vaccine supply is limited, vaccination efforts should focus on administering vaccination to persons at higher risk for medical complications attributable to severe influenza who do not have contraindications. These persons include the following (no hierarchy is implied by order of listing):

- All children aged 6 through 59 months;

- All persons aged $\geq 50$ years;

- Adults and children who have chronic pulmonary (including asthma), cardiovascular (excluding isolated hypertension), renal, hepatic, neurologic, hematologic, or metabolic disorders (including diabetes mellitus);

- Persons who are immunocompromised due to any cause (including but not limited to immunosuppression caused by medications or HIV infection);

- Women who are or will be pregnant during the influenza season;

- Children and adolescents (aged 6 months through 18 years) who are receiving aspirin- or salicylate-containing medications and who might be at risk for experiencing Reye syndrome after influenza virus infection;

- Residents of nursing homes and other long-term care facilities;

- American Indians/Alaska Natives; and 
- Persons who are extremely obese (body mass index $\geq 40$ for adults).

An IIV4 or RIV4 (as appropriate for the recipient's age) is suitable for persons in all risk groups. LAIV4 is not recommended for some populations, including some of these listed groups. Contraindications and precautions to the use of LAIV4 are noted (Table 2).

\section{Persons Who Live with or Care for Persons at Higher Risk for Influenza-Related Complications}

All persons aged $\geq 6$ months without contraindications should be vaccinated annually; however, in addition to persons at higher risk for medical complications attributable to severe influenza, emphasis also should be placed on vaccination of persons who live with or care for those who are at increased risk. When vaccine supply is limited, vaccination efforts should focus on administering vaccination to persons at higher risk for influenza-related complications, as well as persons who live with or care for such persons, including the following:

- Health care personnel, including all paid and unpaid persons working in health care settings who have the potential for exposure to patients or to infectious materials. These personnel might include (but are not limited to) physicians, nurses, nursing assistants, nurse practitioners, physician assistants, therapists, technicians, emergency medical service personnel, dental personnel, pharmacists, laboratory personnel, autopsy personnel, students and trainees, contractual staff, and other persons not directly involved in patient care but who might be exposed to infectious agents (e.g., clerical, dietary, housekeeping, laundry, security, maintenance, administrative, and billing staff and volunteers). ACIP guidance for vaccination of health care personnel has been published previously (44);

- Household contacts (including children aged $\geq 6$ months) and caregivers of children aged $\leq 59$ months (i.e., aged $<5$ years) and adults aged $\geq 50$ years, particularly contacts of children aged $<6$ months; and

- Household contacts (including children aged $\geq 6$ months) and caregivers of persons with medical conditions that put them at higher risk for severe complications from influenza.

Health care personnel and persons who are contacts of persons in these groups (with the exception of contacts of severely immunocompromised persons who require a protected environment) may receive any influenza vaccine that is otherwise indicated. Persons who care for severely immunocompromised persons requiring a protected environment should receive either IIV4 or RIV4. ACIP and the Healthcare Infection Control Practices Advisory Committee (HICPAC) have previously recommended that health care personnel who receive LAIV should avoid providing care for severely immunosuppressed patients requiring a protected environment for 7 days after vaccination and that hospital visitors who have received LAIV should avoid contact with such persons for 7 days after vaccination (45). However, such persons need not be restricted from caring for or visiting less severely immunosuppressed patients.

\section{Influenza Vaccination of Persons with COVID-19}

Experience with influenza vaccination of persons with COVID-19 is limited. Considerations regarding vaccination of persons who have tested positive for COVID-19 or who are in quarantine after an exposure should include whether bringing the recipient into a vaccination setting could expose others to COVID-19, whether the person is acutely ill and the severity of the illness, presence of risk factors for severe influenza illness, the likelihood of being able to vaccinate at a later date, and the desire to avoid confusing postvaccination symptoms with those of COVID-19 illness. In general, those who are in quarantine or isolation should not be brought to a vaccination setting if doing so could expose others to COVID-19. For those who have moderate or severe COVID-19, vaccination should generally be deferred until they have recovered, which is consistent with ACIP General Best Practice Guidelines for Immunization (46). For persons who have mild or asymptomatic COVID-19, further deferral might be considered to avoid confusing COVID-19 illness symptoms with postvaccination reactions. Because recommendations for vaccination of this population might continue to evolve, clinicians should check current CDC guidance (https://www. cdc.gov/vaccines/pandemic-guidance/index.html) for up-todate information.

\section{Children Aged 6 Months Through 8 Years}

Vaccines and dose volumes for children aged 6 through 35 months: Four IIV $4 s$ are approved for ages $\geq 6$ months; one is approved for ages $\geq 2$ years. The appropriate dose volumes for some of these vaccines differ for children aged $<36$ months from those for older children and adults (Table 4). For these vaccines, approved age indications and dose volumes are as follows:

- Afluria Quadrivalent is approved for ages $\geq 6$ months. The approved dose volume for children aged 6 through 35 months is $0.25 \mathrm{~mL}$ per dose. Persons aged $\geq 36$ months ( $\geq 3$ years) should receive $0.5 \mathrm{~mL}$ per dose.

- Fluarix Quadrivalent is approved for ages $\geq 6$ months. The approved dose volume is $0.5 \mathrm{~mL}$ per dose for all persons aged $\geq 6$ months.

- FluLaval Quadrivalent is approved for ages $\geq 6$ months. The approved dose volume is $0.5 \mathrm{~mL}$ per dose for all persons aged $\geq 6$ months. 
TABLE 4. Dose volumes for inactivated influenza vaccines approved for children aged 6 through 35 months* — United States, 2021-22 influenza season

\begin{tabular}{lc}
\hline Trade name (Manufacturer) & $\begin{array}{c}\text { Dose volume for children aged } \\
6 \text { through } 35 \text { mos } \\
(\mu \mathrm{g} \mathrm{HA} \mathrm{per} \mathrm{vaccine} \mathrm{virus)}\end{array}$ \\
\hline Afluria Quadrivalent (Seqirus) & $0.25 \mathrm{~mL}(7.5 \mu \mathrm{g})$ \\
Fluarix Quadrivalent (GlaxoSmithKline) & $0.5 \mathrm{~mL}(15 \mu \mathrm{g})$ \\
FluLaval Quadrivalent (GlaxoSmithKline) & $0.5 \mathrm{~mL}(15 \mu \mathrm{g})$ \\
Fluzone Quadrivalent (Sanofi Pasteur) & $0.25 \mathrm{~mL}(7.5 \mu \mathrm{g})$ \\
& or $0.5 \mathrm{~mL}(15 \mu \mathrm{g})^{\dagger}$ \\
Flucelvax Quadrivalent (Seqirus) & $0.5 \mathrm{~mL}(15 \mu \mathrm{g})^{\S}$ \\
(ages $\geq 2$ yrs only; not approved for ages $^{\S}$ through 23 mos) & \\
\hline
\end{tabular}

Abbreviation: $\mathrm{HA}=$ hemagglutinin.

* For persons aged $\geq 36$ months ( $\geq 3$ years), the dose volume is $0.5 \mathrm{~mL}$ per dose for all inactivated influenza vaccines with the exception of Fluzone High-Dose Quadrivalent (HD-IIV4), which is licensed for persons aged $\geq 65$ years and for which the dose volume is $0.7 \mathrm{~mL}$ per dose.

† Fluzone Quadrivalent is currently approved for ages 6 through 35 months at either $0.25 \mathrm{~mL}$ or $0.5 \mathrm{~mL}$ per dose; however, 0.25 - $\mathrm{mL}$ prefilled syringes are not expected to be available for the 2021-22 season. If a prefilled syringe of Fluzone Quadrivalent is used for a child in this age group, the dose volume will be $0.5 \mathrm{~mL}$ per dose. The $0.5-\mathrm{mL}$ single-dose vials should be accessed for only 1 dose and multidose vials for only 10 doses, regardless of the volume of the doses taken or any remaining volume in the vial. Any vaccine remaining in a vial after the maximum number of doses has been removed should be discarded.

$\S$ As of August 2021, Flucelvax Quadrivalent is approved for ages $\geq 2$ years. The dose volume is $0.5 \mathrm{~mL}$ per dose for all persons aged $\geq 24$ months ( $\geq 2$ years).

- Fluzone Quadrivalent is approved for ages $\geq 6$ months. The approved dose volume for children aged 6 through 35 months is either $0.25 \mathrm{~mL}$ or $0.5 \mathrm{~mL}$ per dose. Persons aged $\geq 36$ months should receive $0.5 \mathrm{~mL}$ per dose.

- Flucelvax Quadrivalent is approved for ages $\geq 2$ years. The approved dose volume is $0.5 \mathrm{~mL}$ per dose for all persons aged $\geq 24$ months ( $\geq 2$ years).

Alternatively, healthy children aged $\geq 24$ months ( $\geq 2$ years) may receive LAIV $4,0.2 \mathrm{~mL}$ intranasally $(0.1 \mathrm{~mL}$ in each nostril). LAIV4 is not recommended for some populations (see Contraindications and Precautions for the Use of LAIV4) (Table 2), and is not approved for children aged $<2$ years. RIV4 is not approved for children aged $<18$ years. High-dose inactivated influenza vaccine (HD-IIV4) and adjuvanted inactivated influenza vaccine (aIIV4) are not approved for persons aged $<65$ years.

Care should be taken to administer an age-appropriate vaccine at the appropriate volume for each dose. For IIV $4 \mathrm{~s}$, the recommended volume may be administered from a prefilled syringe containing the appropriate volume (as supplied by the manufacturer), a single-dose vial, or a multidose vial. Afluria Quadrivalent is approved for children aged 6 through 35 months at $0.25 \mathrm{~mL}$ per dose. Fluzone Quadrivalent is approved for children aged 6 through 35 months at either $0.25 \mathrm{~mL}$ or $0.5 \mathrm{~mL}$ per dose. However, the $0.25-\mathrm{mL}$ prefilled syringe presentation of Fluzone Quadrivalent is not anticipated to be available for the 2021-22 season. If a prefilled syringe of Fluzone Quadrivalent is used for a child in this age group, the dose volume will be $0.5 \mathrm{~mL}$ per dose. Single-dose, $0.5-\mathrm{mL}$ vials of Fluzone Quadrivalent should be used for only 1 dose, and multidose vials for only 10 doses, regardless of the volume of the doses taken or any remaining volume in the vial. Any vaccine remaining in a vial after the maximum number of doses has been removed should be discarded.

Number of doses for children aged 6 months through 8 years: Children aged 6 months through 8 years require 2 doses of influenza vaccine administered a minimum of 4 weeks apart during their first season of vaccination for optimal protection (47-50). Determination of the number of doses needed is based on 1) the child's age at the time of the first dose of 2021-22 influenza vaccine and 2) the number of doses of influenza vaccine received in previous influenza seasons:

- For those aged 6 months through 8 years, the number of doses of influenza vaccine needed for the 2021-22 influenza season is determined as follows (Figure):

- Those who have previously received $\geq 2$ total doses of trivalent or quadrivalent influenza vaccine $\geq 4$ weeks apart before July 1, 2021, require only 1 dose for the 2021-22 season. The 2 previous doses of influenza vaccine do not need to have been administered in the same season or consecutive seasons.

- Those who have not previously received $\geq 2$ doses of trivalent or quadrivalent influenza vaccine $\geq 4$ weeks apart before July 1, 2021, or whose previous influenza vaccination history is unknown, require 2 doses for the 2021-22 season. The interval between the 2 doses should be $\geq 4$ weeks. Two doses are recommended even if the child turns age 9 years between receipt of dose 1 and dose 2.

- Adults and children aged $\geq 9$ years need only 1 dose of influenza vaccine for the 2021-22 season.

\section{Pregnant Women}

Pregnant and postpartum women have been observed to be at higher risk for severe illness and complications from influenza, particularly during the second and third trimesters. Influenza vaccination during pregnancy is associated with reduced risk for respiratory illness and influenza among pregnant and postpartum women, as well as infants during the first several months of life (36-40). ACIP and the American College of Obstetricians and Gynecologists recommend that those who are pregnant or who might be pregnant or postpartum during the influenza season receive influenza vaccine $(51,52)$. Any licensed, recommended, and age-appropriate IIV 4 or RIV4 may be used. LAIV4 should not be used during pregnancy but can be used postpartum. Influenza vaccine can be administered at any time during pregnancy, before and during the influenza season. 
FIGURE. Influenza vaccine dosing algorithm for children aged 6 months through 8 years* - Advisory Committee on Immunization Practices, United States, 2021-22 influenza season

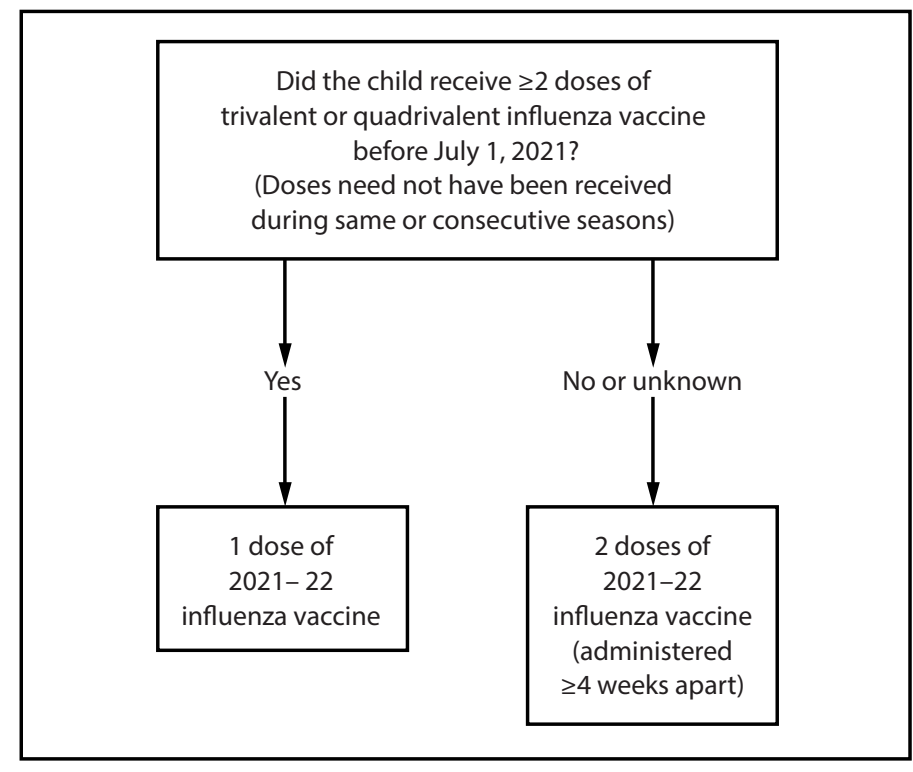

* For children aged 8 years who require 2 doses of vaccine, both doses should be administered even if the child turns age 9 years between receipt of dose 1 and dose 2 .

Although experience with the use of IIVs during pregnancy is substantial, data specifically reflecting administration of influenza vaccines during the first trimester are relatively limited (see Safety of Influenza Vaccines in the supplementary Background Document). Most studies have not noted an association between influenza vaccination and adverse pregnancy outcomes, including spontaneous abortion (53-63). One observational Vaccine Safety Datalink (VSD) study conducted during the 2010-11 and 2011-12 seasons noted an association between receipt of IIV containing influenza $\mathrm{A}(\mathrm{H} 1 \mathrm{~N} 1) \mathrm{pdm} 09$ and risk for spontaneous abortion (miscarriage) in the 28 days after receipt of IIV, when an $\mathrm{H} 1 \mathrm{~N} 1 \mathrm{pdm} 09$-containing vaccine had also been received the previous season (64). However, in a larger VSD follow-up study, IIV was not associated with an increased risk for spontaneous abortion during the 2012-13, 2013-14, and 2014-15 seasons, regardless of previous season vaccination (65).

Substantially less experience exists with more recently licensed IIVs (e.g., quadrivalent and cell culture-based vaccines) during pregnancy as compared with previously available products. For RIV (available as RIV3 from 2013-14 through 2017-18 and as RIV4 since 2017-18), data are limited to reports of pregnancies occurring incidentally during clinical trials, Vaccine Adverse Event Reporting System (VAERS) reports, and pregnancy registries. Pregnancy registries and surveillance studies exist for some products; information can be found in package inserts.

\section{Older Adults}

Because of the vulnerability of older adults to influenzaassociated severe illness, hospitalization, and death, efficacy and effectiveness of influenza vaccines in this population is an area of active research (see Immunogenicity, Efficacy, and Effectiveness of Influenza Vaccines: HD-IIV3, aIIV3, and RIV4 for Older Adults in the Background Document). Comparative studies of vaccine efficacy and effectiveness against laboratory-confirmed influenza outcomes among older adults have focused on Fluzone High-Dose (HD-IIV3) (66), Flublok Quadrivalent (RIV4) (67), and Fluad (aIIV3) (68) (see Table in the Background Document). These studies have evaluated each of these three vaccines compared with standard-dose, nonadjuvanted IIVs (SD-IIVs). HD-IIV3 has been the most extensively studied in this regard, and evidence has accumulated for its superior efficacy and effectiveness compared with SD-IIV3 in this population. For the 2020-21 season, quadrivalent formulations of high-dose (HD-IIV4) and adjuvanted (aIIV4) influenza vaccines were introduced; trivalent formulations of these vaccines will not be available for the 2021-22 season. Data summarizing comparisons of these newer quadrivalent formulations relative to standarddose, nonadjuvanted IIV4 against laboratory-confirmed influenza outcomes are not yet available. Moreover, data from studies comparing the efficacy or effectiveness of HD-IIVs, aIIVs, and RIV4 directly with one another against laboratory-confirmed influenza outcomes among older adults are limited. In comparative safety studies, some injection site and systemic reactions were observed more frequently in older persons vaccinated with HD-IIV3 and aIIV3 compared with nonadjuvanted SD-IIV3 $(69,70)$.

Fluzone High-Dose (HD-IIV3) met prespecified criteria for superior efficacy against laboratory-confirmed influenza compared with that of standard-dose Fluzone (SD-IIV3) in a randomized trial conducted over two influenza seasons (2011-12 and 2012-13) among 31,989 persons aged $\geq 65$ years $(66,71)$. For the primary outcome (prevention of laboratoryconfirmed influenza caused by any viral type or subtype and associated with protocol-defined influenza-like illness [ILI]), the relative efficacy of Fluzone HD-IIV3 compared with Fluzone SD-IIV3 was $24.2 \%(95 \% \mathrm{CI}=9.7 \%-36.5 \%)$. These findings are further supported by results from retrospective studies of data from the Centers for Medicare and Medicaid Services and the Veterans Administration, as well as a clusterrandomized trial of HD-IIV3 compared with SD-IIV among older adults in nursing homes (72-76). A meta-analysis reported that HD-IIV3 provided better protection than SD-IIV3 against ILI (relative vaccine effectiveness $=19.5 \%$; 95\% CI $=8.6 \%-29.0 \%$ ); all-cause hospitalizations (relative 
vaccine effectiveness $=9.1 \% ; 95 \% \mathrm{CI}=2.4 \%-15.3 \%$ ); and hospitalizations due to influenza (relative vaccine effectiveness $=17.8 \% ; 95 \% \mathrm{CI}=8.1 \%-26.5 \%$ ), pneumonia (relative vaccine effectiveness $=24.3 \%$; $95 \% \mathrm{CI}=13.9 \%-$ $33.4 \%$ ), and cardiorespiratory events (relative vaccine effectiveness $=18.2 \%$; 95\% CI $=6.8 \%-28.1 \%)(77)$. For the 2020-21 season, HD-IIV3 was replaced by Fluzone High-Dose Quadrivalent (HD-IIV4). HD-IIV4 exhibited noninferior immunogenicity compared with HD-IIV3 in a randomized trial $(78,79)$; estimates of relative efficacy compared with standard-dose nonadjuvanted IIV4 against laboratory-confirmed influenza outcomes are not available.

In an exploratory analysis of data from a single-season (2014-15) randomized trial conducted among 8,604 adults aged $\geq 50$ years, Flublok Quadrivalent (RIV4) was more efficacious than SD-IIV4 $(67,80)$; however, no claim of superiority was approved for the package insert (80). For the primary outcome (protocol-defined ILI caused by any influenza virus type or subtype and confirmed by reverse transcription-polymerase chain reaction [RT-PCR]), the relative vaccine effectiveness of RIV 4 compared with SD-IIV4 was 30\% (95\% CI $=10 \%-47 \%)$. When restricted to persons aged $\geq 65$ years, the relative vaccine effectiveness of RIV4 was $17 \%(95 \% \mathrm{CI}=-20 \%-43 \%)$.

In an observational study from a single season (2011-12), Fluad (aIIV3) was more effective against laboratory-confirmed influenza than nonadjuvanted SD-IIV3 among adults aged $\geq 65$ years $(\mathrm{N}=227,165$ of whom received aIIV3 and 62 SD-IIV3) (68); the population receiving aIIV3 had a higher proportion of persons in long-term care facilities (81). The relative effectiveness of aIIV3 compared with nonadjuvanted SD-IIV3 was $63 \%(95 \% \mathrm{CI}=4 \%-86 \%)$. In other observational studies, aIIV3 was associated with reduced risk for hospitalization for pneumonia and influenza diagnoses (82) and pneumonia, cerebrovascular, or cardiovascular diagnoses relative to nonadjuvanted IIV 3 in studies of medical record data (83). For the 2021-22 season, Fluad (aIIV3) is no longer expected to be available. The quadrivalent formulation (Fluad Quadrivalent, aIIV4) met prespecified immunogenicity criteria relative to a noninfluenza control vaccine in a randomized trial; although the primary efficacy outcome was not met, the majority of influenza infections during the study were associated with a mismatched $\mathrm{A}(\mathrm{H} 3 \mathrm{~N} 2)$ virus $(84,85)$. In a second randomized study examining immunogenicity of aIIV4 relative to aIIV3, aIIV4 met prespecified noninferiority criteria compared with aIIV3 for all four viruses. When considered individually, aIIV3 and aIIV4 each met criteria for seroconversion and proportion of participants achieving an $\mathrm{HA}$ titer of 1:40 for the influenza $\mathrm{A}(\mathrm{H} 1 \mathrm{~N} 1) \mathrm{pdm} 09$ and $\mathrm{A}(\mathrm{H} 3 \mathrm{~N} 2)$ viruses; however, neither the quadrivalent nor trivalent vaccines met these criteria for the influenza B viruses. This might have been related to the lower likelihood of generating this response in a highly vaccinated population $(86,87)$. Estimates of relative efficacy of aIIV4 compared with nonadjuvanted IIV4 against laboratory-confirmed influenza outcomes are not yet available.

Data reflecting comparisons of HD-IIVs, aIIVs, and RIVs are limited. Retrospective analyses of Centers for Medicare and Medicaid Services (CMS) data from the 2017-18, 2018-19, and 2019-20 influenza seasons, each including 12-13 million persons aged $\geq 65$ years, have compared effectiveness of various vaccine types relative to egg-based, standard-dose, unadjuvanted IIV4 against influenza-associated hospital encounters (hospitalizations and emergency department visits, defined by International Classification of Diseases [ICD] codes) (88-90). These analyses included HD-IIV3, aIIV3, and ccIIV4 for all three seasons; RIV4 was included in the 2019-20 analysis. Compared with egg-based IIV4, for the 2017-18 season, relative effectiveness was noted with ccIIV4 (11.0\%), HD-IIV3 (9.0\%), and aIIV3 (3.9\%); for 2018-19, with aIIV3 (7.7\%) and HD-IIV3 (4.9\%); and for 2019-20, with RIV4 (13.3\%), aIIV3 (8.2\%), and HD-IIV3 (6.8\%).

One postlicensure randomized clinical trial in the United States evaluated the comparative safety of aIIV3 compared with HD-IIV3 in 757 adults aged $\geq 65$ years (378 who received aIIV3 versus 379 who received HD-IIV3) (91). For the primary outcome, the proportion of participants who reported moderate to severe injection site pain that limited or prevented activity after aIIV3 (12 participants [3.2\%]) was noninferior compared with the proportion reporting this outcome following HD-IIV3 (22 participants [5.8\%]). No participant sought medical care for a solicited reaction symptom, and none had a serious adverse event determined by study investigators to be related to vaccine within 43 days after vaccination.

ACIP will continue to review data on the efficacy and effectiveness of influenza vaccines as more information becomes available. No preference is expressed for any one vaccine type. Vaccination should not be delayed if a specific vaccine is not readily available. For persons aged $\geq 65$ years, any age-appropriate IIV 4 formulation (standard dose or high dose, nonadjuvanted or adjuvanted) or RIV4 is an acceptable option.

\section{Immunocompromised Persons}

ACIP recommends that persons with immunocompromising conditions (including but not limited to persons with congenital and acquired immunodeficiency states, persons who are immunocompromised due to medications, and persons with anatomic and functional asplenia) should receive an age-appropriate IIV4 or RIV4. ACIP recommends that LAIV4 not be used for these groups because of the uncertain 
but biologically plausible risk for disease attributable to the live vaccine virus. Use of LAIV4 in persons with these and other conditions is discussed in more detail (see Dosage, Administration, Contraindications, and Precautions) (Table 2).

Immunocompromised states comprise a heterogeneous range of conditions with varying risks for severe infections. In many instances, limited data are available regarding the use of influenza vaccines in the setting of specific immunocompromised states. Timing of vaccination might be a consideration (e.g., vaccinating during some period either before or after an immunocompromising intervention). The Infectious Diseases Society of America has published detailed guidance for the selection and timing of vaccines for persons with specific immunocompromising conditions (92). Immune response to influenza vaccines might be blunted in persons with some conditions, such as persons with congenital immune deficiencies, and persons receiving cancer chemotherapy or immunosuppressive medications.

\section{Persons with a History of Guillain-Barré Syndrome After Influenza Vaccination}

A history of Guillain-Barré syndrome (GBS) within 6 weeks of a previous dose of any type of influenza vaccine is considered a precaution to influenza vaccination (Table 2). Persons who are not at higher risk for severe influenza complications (see Populations at Higher Risk for Medical Complications Attributable to Severe Influenza) and who are known to have experienced GBS within 6 weeks of a previous influenza vaccination generally should not be vaccinated. As an alternative to vaccination, providers might consider using influenza antiviral chemoprophylaxis for these persons (93). However, the benefits of influenza vaccination might outweigh the possible risks for certain persons who have a history of GBS within 6 weeks after receipt of influenza vaccine and who also are at higher risk for severe complications from influenza.

\section{Persons with a History of Egg Allergy}

Most available influenza vaccines, with the exceptions of RIV4 (Flublok Quadrivalent, licensed for those aged $\geq 18$ years) and ccIIV4 (Flucelvax Quadrivalent, licensed for those aged $\geq 2$ years), are prepared by propagation of virus in embryonated eggs and might contain trace amounts of egg proteins, such as ovalbumin. For persons who report a history of egg allergy, ACIP recommends the following:

- Persons with a history of egg allergy who have experienced only urticaria (hives) after exposure to egg should receive influenza vaccine. Any licensed, recommended influenza vaccine (i.e., any IIV4, RIV4, or LAIV4) that is otherwise appropriate for the recipient's age and health status can be used.
- Persons who report having had reactions to egg involving symptoms other than urticaria (e.g., angioedema or swelling, respiratory distress, lightheadedness, or recurrent vomiting) or who required epinephrine or another emergency medical intervention can similarly receive any licensed, recommended influenza vaccine (i.e., any IIV4, RIV4, or LAIV4) that is otherwise appropriate for their age and health status. If a vaccine other than ccIIV4 or RIV4 is used, the selected vaccine should be administered in an inpatient or outpatient medical setting (including but not necessarily limited to hospitals, clinics, health departments, and physician offices). Vaccine administration should be supervised by a health care provider who is able to recognize and manage severe allergic reactions.

All vaccine providers should be familiar with their office emergency plan and be certified in cardiopulmonary resuscitation (46). No postvaccination observation period is recommended specifically for egg-allergic persons. However, ACIP recommends that vaccine providers consider observing patients (seated or supine) for 15 minutes after administration of any vaccine to decrease the risk for injury should syncope occur (46).

\section{Persons with Previous Allergic Reactions to Influenza Vaccines}

As is the case for all vaccines, influenza vaccines contain various components that might cause allergic and anaphylactic reactions. Most influenza vaccine package inserts list among contraindications to their use a history of previous severe allergic reaction (e.g., anaphylaxis) to any component of the vaccine or to a previous dose of any influenza vaccine. For ccIIV4 and RIV4, history of a severe allergic reaction to any vaccine component is listed as a contraindication; no labeled contraindication is specified for history of allergic reaction to any other influenza vaccine. However, severe allergic reactions, although rare, can occur after influenza vaccination, even among persons with no previous reactions or known allergies. Although vaccine components can be found in package inserts, identifying the causative component without further evaluation (i.e., through evaluation and testing for specific allergies) can be difficult. Severe allergic reactions after vaccination with an RIV have been reported to VAERS, including some that have occurred among persons reporting previous allergic reactions to egg or to influenza vaccines and that might represent a predisposition to development of allergic manifestations in affected persons (94-96). Because these rare but severe allergic reactions can occur, ACIP recommends the following for persons with a history of severe allergic reaction to a previous dose of an influenza vaccine (Table 3): 
- For egg-based IIV4s and LAIV4: A history of severe allergic reaction (e.g., anaphylaxis) to any influenza vaccine (i.e., any egg-based IIV, ccIIV, RIV, or LAIV of any valency) is a contraindication to future receipt of all egg-based IIV $4 \mathrm{~s}$ and LAIV4. Each individual egg-based IIV4 and LAIV4 is also contraindicated for persons who have had a severe allergic reaction (e.g., anaphylaxis) to any component of that vaccine.

- For ccIIV4:

- A history of a severe allergic reaction (e.g., anaphylaxis) to any egg-based IIV, RIV, or LAIV of any valency is a precaution to the use of ccIIV4. If ccIIV4 is administered in such instances, vaccination should occur in an inpatient or outpatient medical setting and should be supervised by a health care provider who is able to recognize and manage severe allergic reactions. Providers also can consider consultation with an allergist to help determine the vaccine component responsible for the allergic reaction.

- A history of a severe allergic reaction (e.g., anaphylaxis) to any ccIIV of any valency or to any component of ccIIV4 is a contraindication to future receipt of ccIIV4.

- For RIV4:

$\circ$ A history of a severe allergic reaction (e.g., anaphylaxis) to any egg-based IIV, ccIIV, or LAIV of any valency is a precaution to the use of RIV4. If RIV4 is administered in such instances, vaccination should occur in an inpatient or outpatient medical setting and should be supervised by a health care provider who is able to recognize and manage severe allergic reactions. Providers can also consider consulting with an allergist to help determine the vaccine component responsible for the allergic reaction.

- A history of a severe allergic reaction (e.g., anaphylaxis) to any RIV of any valency or to any component of RIV4 is a contraindication to future receipt of RIV4

\section{Vaccination Issues for Travelers}

In temperate climate regions of the Northern and Southern Hemispheres, influenza activity is seasonal, occurring approximately from October-May in the Northern Hemisphere and April-September in the Southern Hemisphere. In the tropics, influenza might occur throughout the year. Travelers can be exposed to influenza when traveling to an area where influenza is circulating or when traveling as part of large tourist groups (e.g., on cruise ships) that include persons from areas of the world where influenza viruses are circulating (97-100).

Travelers who want to reduce their risk for influenza should consider influenza vaccination, preferably at least 2 weeks before departure. In particular, persons who live in the United
States and are at higher risk for influenza complications and who were not vaccinated with influenza vaccine during the previous Northern Hemisphere fall or winter should consider receiving influenza vaccination before departure if they plan to travel to the tropics, to the Southern Hemisphere during the Southern Hemisphere influenza season (April-September), or with organized tourist groups or on cruise ships to any location. Persons at higher risk who received the previous season's influenza vaccine before travel should consult with their health care provider to discuss the risk for influenza and other travel-related diseases before embarking on travel during the summer. All persons (regardless of risk status) who are vaccinated in preparation for travel before the upcoming influenza season's vaccine is available should receive the current vaccine the following fall or winter.

Influenza vaccine formulated for the Southern Hemisphere might differ in viral composition from the Northern Hemisphere vaccine. For persons traveling to the Southern Hemisphere during the Southern Hemisphere influenza season, receipt of a current U.S.-licensed Southern Hemisphere formulation influenza vaccine before departure might be reasonable but might not be feasible because of limited access to or unavailability of Southern Hemisphere formulations in the United States. Most Southern Hemisphere influenza vaccine formulations are not licensed in the United States, and they are generally are not commercially available. More information on influenza vaccines and travel is available at https://wwwnc.cdc. gov/travel/diseases/influenza-seasonal-zoonotic-and-pandemic.

\section{Use of Influenza Antiviral Medications}

Administration of IIV4 or RIV4 to persons receiving influenza antiviral medications for treatment or chemoprophylaxis of influenza is acceptable. Data concerning vaccination with LAIV4 in the setting of influenza antiviral use are not available. However, influenza antiviral medications might interfere with the action of LAIV4 because this vaccine contains live influenza viruses.

The package insert for LAIV4 notes that antiviral agents might reduce the effectiveness of the vaccine if given within the interval from 48 hours before to 14 days after vaccination (101). However, the newer influenza antivirals peramivir and baloxavir have longer half-lives than oseltamivir and zanamivir, with approximately 20 hours for peramivir (102) and 79 hours for baloxavir (103), and could conceivably interfere with the replication of LAIV4 if administered $>48$ hours before vaccination. Potential interactions between influenza antivirals and LAIV4 have not been studied, and the ideal intervals between administration of these medications and LAIV4 are not known. Assuming a period of at least 5 half-lives for substantial decline in drug levels (104), it is reasonable to 
assume that peramivir might interfere with the mechanism of LAIV4 if administered from 5 days before through 2 weeks after vaccination, and baloxavir might interfere if administered from 17 days before through 2 weeks after vaccination. The interval between influenza antiviral receipt and LAIV4 for which interference might occur could be further prolonged in the presence of medical conditions that delay medication clearance (e.g., renal insufficiency). Persons who receive these medications during these periods before or after receipt of LAIV4 should be revaccinated with another appropriate influenza vaccine (e.g., IIV4 or RIV4).

\section{Administration of Influenza Vaccines with Other Vaccines}

With regard to administration of influenza vaccines with vaccines other than COVID-19 vaccines, guidance is as follows. IIV4s and RIV4 may be administered simultaneously or sequentially with other inactivated vaccines or live vaccines. Injectable vaccines that are given concomitantly should be administered at separate anatomic sites. LAIV4 can be administered simultaneously with other live or inactivated vaccines. However, if two live vaccines are not given simultaneously, then after administration of one live vaccine (such as LAIV4), at least 4 weeks should pass before another live vaccine is administered (46).

Guidance concerning administration of COVID-19 vaccines with other vaccines (https://www.cdc.gov/vaccines/covid-19/ clinical-considerations/covid-19-vaccines-us.html) indicates that these vaccines may be given with other vaccines, including influenza vaccines. No data are yet available concerning coadministration of U.S.-authorized COVID-19 vaccines and influenza vaccines. Providers should be aware of the potential for increased reactogenicity with coadministration and should consult the CDC guidance as more information becomes available. If administered simultaneously, COVID-19 vaccines and influenza vaccines that might be more likely to cause a local reaction (e.g., aIIV4 or HD-IIV4) should be administered in different limbs, if possible.

Relatively limited data are available on the concomitant administration of influenza vaccines with other vaccines. Studies of live attenuated zoster vaccine and IIV3 (105) or IIV4 (106) among persons aged $\geq 50$ years noted similar antibody responses whether the two vaccines were administered concomitantly or 4 weeks apart. In some studies, reduced responses have been noted to 13-valent pneumococcal conjugate vaccine (PCV13) $(107,108)$, tetanus antigens (109), and pertussis antigens (109) when coadministered with IIV3 to adults; in most instances, the clinical significance of this is uncertain. Simultaneous administration of IIV4 and 23-valent pneumococcal polysaccharide vaccine (PPSV23) to persons aged $\geq 65$ years was associated with lower seroprotection rates to one influenza B antigen at 4-6 weeks postvaccination as compared with sequential administration 2 weeks apart; seroprotection was not significantly different between the two groups for any of the four influenza antigens at 6 months postvaccination (110). Reassuring safety profiles have been noted for simultaneous administration of IIVs with live attenuated zoster vaccine $(105,106)$; PCV13 $(107,108)$; PPSV23 (110,111); tetanus toxoid, reduced diphtheria toxoid, and acellular pertussis (Tdap) vaccine among adults (109); and Tdap in pregnancy (112). Although increased prevalence of injection site or systemic adverse reactions has been noted with concurrent administration in some of these studies, these symptoms have generally been reported to be mild or moderate.

Among children aged 6 through 23 months, coadministration of IIV3 and PCV13 was associated with increased risk for fever on the day of vaccination and the day after (i.e., days $0-1$ postvaccination) in an observational study conducted during the 2011-12 season (113). A randomized clinical trial during the 2017-18 influenza season suggested that delaying IIV 4 administration by 2 weeks in children receiving DTaP and PCV13 did not reduce fever prevalence after vaccination (114). Increased risk for febrile seizures in this age group has been noted within days $0-1$ after coadministration of IIV with PCV7, PCV13, or diphtheria and tetanus toxoids and acellular pertussis (DTaP) vaccines during the 2006-07 through 2010-11 seasons (115) and with PCV13 during the 2014-15 season (116). Although concerning to parents, most febrile seizures are brief and have a good prognosis (117). After considering the risks and benefits, no changes in the recommendations for administration of these vaccines were made, and these vaccines can be given concomitantly. Surveillance of febrile seizures is ongoing through VAERS, and the VSD annual influenza safety surveillance includes monitoring for seizures after vaccinations. Studies of concomitant administration of LAIV with other vaccines are limited. Concurrent administration of LAIV3 with measles, mumps, and rubella (MMR) and varicella vaccine to children was not associated with diminished immunogenicity to antigens in any of the vaccines in one study (118); diminished response to rubella was observed in another study examining coadministration of LAIV3 and MMR (119). No safety concerns were noted in these studies.

In recent years, several vaccines containing nonaluminum adjuvants have been licensed for use in the United States for the prevention of various infectious diseases. These include AS0 $1_{B}$ (in Shingrix, recombinant zoster subunit vaccine) (120), MF59 (in Fluad Quadrivalent [aIIV4]) (121), and cytosine phosphoguanine oligodeoxynucleotide (in Heplisav-B, a recombinant hepatitis B surface antigen vaccine) (122). Data are limited regarding coadministration of these vaccines with other adjuvanted or nonadjuvanted vaccines, including COVID-19 vaccines. Coadministration of Shingrix with nonadjuvanted 
IIV4 has been studied; no evidence of decreased immunogenicity or safety concerns were noted (123). The immunogenicity and safety of simultaneous or sequential administration of two nonaluminum-adjuvant-containing vaccines has not been evaluated, and the ideal interval between such vaccines when given sequentially is not known. In the study of Shingrix and IIV4 (123), most reactogenicity symptoms resolved within 4 days. Because of the limited data on the safety of simultaneous administration of two or more vaccines containing nonaluminum adjuvants and the availability of nonadjuvanted influenza vaccine options, selection of a nonadjuvanted influenza vaccine may be considered in situations in which influenza vaccine and another vaccine containing a nonaluminum adjuvant are to be administered concomitantly. However, influenza vaccination should not be delayed if a specific vaccine is not available. As recommended for all vaccines, vaccines with nonaluminum adjuvants should be administered at separate anatomic sites from other vaccines that are given concomitantly (46).

\section{Influenza Vaccine Composition and Available Vaccines}

\section{Influenza Vaccine Composition for the 2021-22 Season}

All influenza vaccines licensed in the United States will contain components derived from influenza viruses antigenically similar to those recommended by FDA (https://www.fda.gov/ advisory-committees/advisory-committee-calendar/vaccinesand-related-biological-products-advisory-committee-march-52021-meeting-announcement\#event-information). Influenza vaccines expected to be available in the United States for the 2021-22 season will be quadrivalent vaccines. For the 2021-22 season, U.S. egg-based influenza vaccines (i.e., vaccines other than ccIIV4 and RIV4) will contain HA derived from

- an influenza A/Victoria/2570/2019(H1N1)pdm09-like virus;

- an influenzaA/Cambodia/e0826360/2020 (H3N2)-like virus;

- an influenza B/Washington/02/2019 (Victoria lineage)like virus; and

- an influenza B/Phuket/3073/2013 (Yamagata lineage)like virus.

For the 2021-22 season, U.S. cell culture-based inactivated (ccIIV4) and recombinant (RIV4) influenza vaccines will contain HA derived from

- an influenzaA/Wisconsin/588/2019(H1N1)pdm09-like virus;

- an influenzaA/Cambodia/e0826360/2020 (H3N2)-like virus;

- an influenza B/Washington/02/2019 (Victoria lineage)like virus; and

- an influenza B/Phuket/3073/2013 (Yamagata lineage)-like virus.

\section{Vaccines Available for the 2021-22 Season}

Various influenza vaccines will be available for the 2021-22 season (Table 1). For many vaccine recipients, more than one type or brand of vaccine might be appropriate within approved indications and ACIP recommendations. A licensed influenza vaccine that is appropriate for the recipient's age and health status should be used. Specific age indications for licensed influenza vaccines are summarized (Table 1); current prescribing information should be consulted for authoritative, up-to-date information. Overall contraindications and precautions for the different types of influenza vaccines are summarized (Table 2), including allergy-specific contraindications and precautions (Table 3), as are dose volumes (Table 4).

Not all influenza vaccines are likely to be uniformly available in any given practice setting or geographic locality. Vaccination should not be delayed to obtain a specific product when an appropriate one is already available. Within these guidelines and approved indications, ACIP makes no preferential recommendation for the use of any one influenza vaccine over another when more than one licensed, recommended, and age-appropriate vaccine is available.

Since the publication of the previous season's guidance, FDA has approved a labeling change for Flucelvax Quadrivalent (see Recent Influenza Vaccine Labeling Changes). Additional new licensures and changes to FDA-approved labeling might occur after publication of this report. As these changes occur and new vaccines become available, they will be reflected in the online version of Table 1, available at https://www.cdc.gov/ flu/professionals/acip/2021-2022/acip-table.htm.

\section{Dosage, Administration, Contraindications, and Precautions}

\section{Quadrivalent Inactivated Influenza Vaccines (IIV4s)}

Available vaccines: As in recent seasons, various inactivated influenza vaccines (IIVs) are expected to be available for 2021-22 (Table 1); all are expected to be quadrivalent (IIV4s). Certain IIV4s are licensed for persons as young as age 6 months. However, licensed age indications differ for different products. Moreover, for some IIV4s, the dose volume for children aged 6 through 35 months differs from that for older children and adults (Table 4). Care should be taken to administer the appropriate dose volume of an age-appropriate vaccine to each recipient.

Standard-dose, nonadjuvanted IIV $4 \mathrm{~s}$ contain $15 \mu \mathrm{g}$ of HA per vaccine virus in a $0.5-\mathrm{mL}$ dose $(7.5 \mu \mathrm{g}$ of HA per vaccine virus in a $0.25-\mathrm{mL}$ dose). For 2021-22, this category is expected to include five different vaccines (Table 1). Four of these are egg-based vaccines, and one is a cell culture-based 
vaccine. Egg-based and cell culture-based vaccines differ in the substrate in which reference vaccine viruses supplied to the manufacturer are propagated in quantities sufficient to produce the needed number of doses of vaccine. The egg-based IIV4s, Afluria Quadrivalent (124), Fluarix Quadrivalent (125), FluLaval Quadrivalent (126), and Fluzone Quadrivalent (127), are approved for persons aged $\geq 6$ months. The cell culturebased IIV4, Flucelvax Quadrivalent (ccIIV4), is approved for persons aged $\geq 2$ years. For the manufacture of ccIIV4, the influenza vaccine viruses are propagated in Madin-Darby canine kidney cells instead of eggs (21).

Two additional IIV4s that will be available for the 2021-2022 season are approved for persons aged $\geq 65$ years. These vaccines are egg based. Quadrivalent high-dose influenza vaccine (Fluzone High-Dose Quadrivalent; HD-IIV4) contains $60 \mu \mathrm{g}$ of HA per vaccine virus $(240 \mu \mathrm{g}$ total) in a $0.7-\mathrm{mL}$ dose (128). Adjuvanted inactivated influenza vaccine (Fluad Quadrivalent; aIIV4) contains $15 \mu \mathrm{g}$ of HA per vaccine virus (60 $\mu \mathrm{g}$ total) and MF59 adjuvant (121).

Dosage and administration: Most, but not all, standarddose unadjuvanted IIV4s are approved for children as young as age 6 months. One exception, Flucelvax Quadrivalent, is approved for persons aged $\geq 2$ years. Some of these IIV $4 s$ are approved at different dose volumes for very young children than for older children and adults. Care should be taken to administer an age-appropriate vaccine at the approved dose volume for each needed dose (see Vaccines and Dose Volumes for Children Aged 6 Through 35 Months) (Tables 1 and 4):

- Afluria Quadrivalent is approved for ages $\geq 6$ months. The approved dose volume for children aged 6 through 35 months is $0.25 \mathrm{~mL}$ per dose. Persons aged $\geq 36$ months ( $\geq 3$ years) should receive $0.5 \mathrm{~mL}$ per dose.

- Fluarix Quadrivalent is approved for ages $\geq 6$ months. The approved dose volume is $0.5 \mathrm{~mL}$ per dose for all persons aged $\geq 6$ months.

- FluLaval Quadrivalent is approved for ages $\geq 6$ months. The approved dose volume is $0.5 \mathrm{~mL}$ per dose for all persons aged $\geq 6$ months.

- Fluzone Quadrivalent is approved for ages $\geq 6$ months. The approved dose volume for children aged 6 through 35 months is either $0.25 \mathrm{~mL}$ or $0.5 \mathrm{~mL}$ per dose. Persons aged $\geq 36$ months ( $\geq 3$ years) should receive $0.5 \mathrm{~mL}$ per dose.

- Flucelvax Quadrivalent is approved for ages $\geq 2$ years. The approved dose volume is $0.5 \mathrm{~mL}$ per dose for all persons aged $\geq 24$ months ( $\geq 2$ years).

Care should be taken to administer the appropriate dose volume for the particular vaccine. If prefilled syringes are not available, the appropriate volume can be administered from a single-dose or multidose vial. If a $0.5-\mathrm{mL}$ single-dose vial is used for a $0.25-\mathrm{mL}$ dose for a child aged 6 through 35 months, only half the vial volume should be administered, and the remaining half should be discarded. Of note, dose volume is distinct from the number of doses. Children in this age group who require 2 doses for 2021-22 (see Children Aged 6 Months through 8 Years) (Figure) need 2 separate doses administered $\geq 4$ weeks apart, regardless of the specific IIV 4 used and volume given for each dose.

For children aged 36 months ( 3 years) through 17 years and adults aged $\geq 18$ years, the dose volume for IIV $4 \mathrm{~s}$ is $0.5 \mathrm{~mL}$ per dose, with the exception of Fluzone High-Dose Quadrivalent (HD-IIV4, licensed for persons aged $\geq 65$ years), for which the correct volume is $0.7 \mathrm{~mL}$ per dose. If a smaller vaccine dose (e.g., $0.25 \mathrm{~mL}$ ) is inadvertently administered to a person aged $\geq 36$ months, the remaining volume needed to make a full dose should be administered during the same vaccination visit or, if measuring the needed remaining volume is a challenge, administering a repeat dose at the full volume is acceptable. If the error is discovered later (after the recipient has left the vaccination setting), a full dose should be administered as soon as the recipient can return. Vaccination with a formulation approved for adult use should be counted as a single dose if inadvertently administered to a child.

IIV $4 s$ are administered intramuscularly (IM). For adults and older children, the deltoid is the preferred site. Infants and younger children should be vaccinated in the anterolateral thigh. Additional specific guidance regarding site selection and needle length for IM injection is provided in the ACIP General Best Practice Guidelines for Immunization (46).

One IIV4, Afluria Quadrivalent, is licensed for IM injection via the PharmaJet Stratis jet injector for persons aged 18 through 64 years (124). Persons in this age group may receive Afluria Quadrivalent via either needle and syringe or this specific jet injection device. Children aged 6 months through 17 years and adults aged $\geq 65$ years should receive this vaccine by needle and syringe only. No other IIV $4 s$ are licensed for administration by jet injector.

Contraindications and precautions for the use of IIV4s: Manufacturer package inserts and updated CDC and ACIP guidance should be consulted for information on contraindications and precautions for individual influenza vaccines. Each IIV, whether egg based or cell culture based, has a labeled contraindication for persons with a history of a severe allergic reaction to any component of that vaccine (Table 2). Although egg is a component of all IIV4s other than ccIIV4, ACIP makes specific recommendations for the use of influenza vaccine for persons with egg allergy (see Persons with a History of Egg Allergy). All egg-based IIV4s are contraindicated in persons who have had a severe allergic reaction (e.g., anaphylaxis) to a previous dose of any influenza vaccine (any egg-based IIV, ccIIV, RIV, or LAIV of any valency). Use of ccIIV4 is contraindicated in persons 
who have had a severe allergic reaction (e.g., anaphylaxis) to any ccIIV of any valency; a history of severe allergic reaction (e.g., anaphylaxis) to any other influenza vaccine (i.e., any egg-based IIV, RIV, or LAIV of any valency) is a precaution to the use of ccIIV4 (see Previous Allergic Reactions to Influenza Vaccines) (Tables 2 and 3). If ccIIV4 is administered in such an instance, vaccination should occur in an inpatient or outpatient medical setting and should be supervised by a health care provider who is able to recognize and manage severe allergic reactions. Providers can also consider consulting with an allergist to help identify the vaccine component responsible for the reaction. Information about vaccine components can be found in the package inserts for each vaccine. Prophylactic use of antiviral agents is an option that can be considered for preventing influenza among persons who cannot receive vaccine, particularly for those who are at higher risk for medical complications attributable to severe influenza (93).

Moderate or severe acute illness with or without fever is a general precaution for vaccination (46). A history of GBS within 6 weeks after receipt of a previous dose of influenza vaccine is considered a precaution for the use of all influenza vaccines (Table 2).

\section{Quadrivalent Recombinant Influenza Vaccine (RIV4)}

Available vaccines: One recombinant influenza vaccine, Flublok Quadrivalent (RIV4), is expected to be available during the 2021-22 influenza season. RIV4 is approved for persons aged $\geq 18$ years. This vaccine contains recombinant $H A$ produced in an insect cell line using genetic sequences from cell-derived influenza viruses and is manufactured without the use of influenza viruses or eggs $(80)$. No preference is expressed for RIV4 versus other influenza vaccines used within specified indications.

Dosage and administration: RIV4 is administered by IM injection via needle and syringe. A $0.5-\mathrm{mL}$ dose contains $45 \mu \mathrm{g}$ of HA derived from each vaccine virus (180 $\mu \mathrm{g}$ total).

Contraindications and precautions for the use of RIV4: RIV4 is contraindicated in persons who have had a severe allergic reaction (e.g., anaphylaxis) to a previous dose of any RIV of any valency or any component of RIV4. A history of a severe allergic reaction (e.g., anaphylaxis) to any other influenza vaccine (i.e., any egg-based IIV, ccIIV, or LAIV of any valency) is a precaution to the use of RIV4. If RIV4 is administered in such an instance, vaccination should occur in an inpatient or outpatient medical setting and should be supervised by a health care provider who is able to recognize and manage severe allergic reactions. Providers can also consider consulting with an allergist to help identify the vaccine component responsible for the reaction. Moderate or severe acute illness with or without fever is a general precaution for vaccination (46). A history of GBS within 6 weeks after receipt of a previous dose of influenza vaccine is considered a precaution for the use of all influenza vaccines (Table 2). RIV4 is not licensed for children aged $<18$ years.

\section{Quadrivalent Live Attenuated Influenza Vaccine (LAIV4)}

Available vaccines: One live attenuated influenza vaccine, FluMist Quadrivalent (LAIV4), is expected to be available during the 2021-22 influenza season. LAIV4 is approved for persons aged 2 through 49 years. LAIV4 contains live attenuated influenza viruses that are propagated in eggs. These viruses are cold adapted (so that they replicate efficiently at $25^{\circ} \mathrm{C}$ ) and temperature sensitive (so that their replication is restricted at higher temperatures, $39^{\circ} \mathrm{C}$ for influenza $\mathrm{A}$ viruses and $37^{\circ} \mathrm{C}$ for influenza $\mathrm{B}$ viruses). These viruses replicate in the nasopharynx, which is necessary to promote an immune response (101). No preference is expressed for LAIV4 versus other influenza vaccines used within specified indications.

Dosage and administration: LAIV4 is administered intranasally using the supplied prefilled, single-use sprayer containing $0.2 \mathrm{~mL}$ of vaccine. Approximately $0.1 \mathrm{~mL}$ (i.e., half the total sprayer contents) is sprayed into the first nostril while the recipient is in the upright position. An attached dose-divider clip is removed from the sprayer to permit administration of the second half of the dose into the other nostril. If the recipient sneezes immediately after administration, the dose should not be repeated. However, if nasal congestion is present that might impede delivery of the vaccine to the nasopharyngeal mucosa, deferral of administration should be considered until resolution of the illness, or another appropriate vaccine should be administered instead. Each total dose of $0.2 \mathrm{~mL}$ contains 10 $0^{6.5-7.5}$ fluorescent focus units of each vaccine virus (101).

Contraindications and precautions for the use of LAIV4: Conditions considered by ACIP to be contraindications and precautions to the use of LAIV4 are summarized (Table 2). These include two labeled contraindications that appear in the package insert (101) and other conditions for which there is uncertain but biologically plausible potential risk associated with live viruses or limited data for use of LAIV.

Contraindications to use of LAIV4 include the following:

- Severe allergic reaction (e.g., anaphylaxis) to any component of the vaccine or to a previous dose of any influenza vaccine (i.e., any egg-based IIV, ccIIV, RIV, or LAIV of any valency; a labeled contraindication noted in the package insert). However, ACIP makes an exception for allergy to egg (see Persons with a History of Egg Allergy);

- Children and adolescents receiving concomitant aspirin or salicylate-containing medications (Table 2), because of the potential risk for Reye syndrome (a labeled contraindication noted in the package insert); 
- Children aged 2 through 4 years who have received a diagnosis of asthma or whose parents or caregivers report that a health care provider has told them during the preceding 12 months that their child had wheezing or asthma or whose medical record indicates a wheezing episode has occurred during the preceding 12 months;

- Children and adults who are immunocompromised due to any cause, including but not limited to immunosuppression caused by medications, congenital or acquired immunodeficiency states, HIV infection, anatomic asplenia, or functional asplenia (such as that due to sickle cell anemia);

- Close contacts and caregivers of severely immunosuppressed persons who require a protected environment;

- Pregnancy;

- Persons with active communication between the cerebrospinal fluid (CSF) and the oropharynx, nasopharynx, nose, or ear or any other cranial CSF leak;

- Persons with cochlear implants, because of the potential for CSF leak, which might exist for some period after implantation (providers might consider consulting with a specialist concerning the risk for persistent CSF leak if an age-appropriate inactivated or recombinant vaccine cannot be used); and

- Receipt of influenza antiviral medication within the previous 48 hours for oseltamivir and zanamivir, previous 5 days for peramivir, and previous 17 days for baloxavir. The interval between influenza antiviral receipt and LAIV4 for which interference might potentially occur might be further prolonged in the presence of medical conditions that delay medication clearance (e.g., renal insufficiency).

Precautions for use of LAIV4 include the following:

- Moderate or severe acute illness with or without fever;

- History of GBS within 6 weeks after receipt of any influenza vaccine;

- Asthma in persons aged $\geq 5$ years; and

- Other underlying medical condition (other than those listed under contraindications) that might predispose to complications after wild-type influenza virus infection (e.g., chronic pulmonary, cardiovascular [except isolated hypertension], renal, hepatic, neurologic, hematologic, or metabolic disorders [including diabetes mellitus]).

\section{Recent Influenza Vaccine Labeling Changes}

\section{Flucelvax Quadrivalent}

Since the publication of the 2020-21 ACIP influenza vaccine statement, there has been a labeling change for Flucelvax Quadrivalent (ccIIV4). Flucelvax Quadrivalent was initially approved in 2016 for persons aged $\geq 4$ years. Approval for ages $\geq 18$ years was based upon a randomized immunogenicity and safety trial which compared Flucelvax Quadrivalent with the previously approved trivalent formulation of Flucelvax (ccIIV3), which had previously been licensed for ages $\geq 18$ years on the basis of data from a randomized clinical efficacy trial. Approval for ages 4 through 17 years was also based on immunogenicity and safety data compared with ccIIV3, with a postmarketing requirement to conduct a clinical efficacy study (129). In March 2021, FDA approved Flucelvax Quadrivalent for ages $\geq 2$ years on the basis of a randomized clinical efficacy trial conducted among 4,514 children aged $\geq 2$ through $<18$ years over three influenza seasons (Southern Hemisphere 2017 and Northern Hemisphere 2017-18 and 2018-19) (130). In this study, children were randomized 1:1 to receive ccIIV4 or a noninfluenza control vaccine (meningococcal serogroup ACWY conjugate vaccine). Overall vaccine efficacy was $54.6 \%$ (95\% CI $=45.7-62.1)$ against CDC-defined ILI associated with RT-PCR-confirmed or culture-confirmed influenza due to all viral strains. Efficacy against culture-confirmed influenza was $60.8 \%(95 \% \mathrm{CI}=51.3-68.5)$ for all viral strains and $63.6 \%$ $(95 \% \mathrm{CI}=53.6-71.5)$ for matched viral strains (21). Frequencies of serious adverse events and most systemic and local solicited adverse reactions were similar between the two study groups, and most solicited reactions were mild or moderate $(21,130)$. With this labeling change, Flucelvax Quadrivalent is now approved for persons aged $\geq 2$ years.

\section{Storage and Handling of Influenza Vaccines}

In all instances, approved manufacturer packaging information should be consulted for authoritative guidance concerning storage and handling of specific influenza vaccines. In general, influenza vaccines should be protected from light and stored at temperatures that are recommended in the package insert. Recommended storage temperatures are generally $36^{\circ} \mathrm{F}-46^{\circ} \mathrm{F}\left(2^{\circ} \mathrm{C}-8^{\circ} \mathrm{C}\right)$ and should be maintained at all times with adequate refrigeration and temperature monitoring. Vaccine that has frozen should be discarded. Specific recommendations for appropriate refrigerators and temperature monitoring equipment can be found in the Vaccine Storage and Handling Toolkit, available at https:// www.cdc.gov/vaccines/hcp/admin/storage/toolkit/index.html.

Vaccines should not be used beyond the expiration date on the label. In addition to the expiration date, multidose vials also might have a Beyond Use Date (BUD), which specifies the number of days the vaccine can be kept once first accessed. Once accessed for the first dose, multidose vials should not 
be used after the BUD. If no BUD is provided, then the listed expiration date is to be used. Multidose vials should be returned to recommended storage conditions between uses. Package information might also specify a maximum number of doses contained in multidose vials (regardless of remaining volume). No more than the specified number of doses should be removed, and any remainder should be discarded. Singledose vials should not be accessed for more than 1 dose. For information on permissible temperature excursions and other departures from recommended storage and handling conditions that are not discussed in the package labeling, contact the manufacturer.

\section{Additional Sources of Information Regarding Influenza and Influenza Vaccines}

\section{Influenza Surveillance, Prevention, and Control}

Updated information regarding influenza surveillance, detection, prevention, and control is available at https:// www.cdc.gov/flu. U.S. surveillance data are updated weekly throughout the year on FluView (https://www.cdc.gov/flu/ weekly) and FluView Interactive (https://www.cdc.gov/flu/ weekly/fluviewinteractive.htm). In addition, periodic updates regarding influenza are published in MMWR (https://www. cdc.gov/mmwr/index.html). Additional information regarding influenza and influenza vaccines can be obtained from CDCINFO by calling 1-800-232-4636. State and local health departments should be consulted about availability of influenza vaccines, access to vaccination programs, information related to state or local influenza activity, reporting of influenza outbreaks and influenza-related pediatric deaths, and advice concerning outbreak control.

\section{Vaccine Adverse Event Reporting System}

The National Childhood Vaccine Injury Act of 1986 requires health care providers to report any adverse event listed by the vaccine manufacturer as a contraindication to future doses of the vaccine or any adverse event listed in the VAERS Table of Reportable Events Following Vaccination (https://vaers.hhs.gov/docs/VAERS_ Table_of_Reportable_Events_Following_Vaccination.pdf) that occurs within the specified period after vaccination. In addition to mandated reporting, health care providers are encouraged to report any clinically significant adverse event after vaccination to VAERS. Information on how to report a vaccine adverse event is available at https://vaers.hhs.gov/index.html.

\section{National Vaccine Injury Compensation Program}

The National Vaccine Injury Compensation Program (VICP), established by the National Childhood Vaccine Injury Act of 1986, as amended, provides a mechanism through which compensation can be paid on behalf of a person determined to have been injured or to have died as a result of receiving a vaccine covered by VICP. The Vaccine Injury Table (https://www.hrsa. gov/sites/default/files/vaccinecompensation/vaccineinjurytable. pdf) lists the vaccines covered by VICP and the associated injuries and conditions (including death) that might receive a legal presumption of causation. If the injury or condition is not in the Table or does not occur within the specified period in the Table, persons must prove that the vaccine caused the injury or condition. Eligibility for compensation is not affected by whether a covered vaccine is used off-label or inconsistently with recommendations. To be eligible for compensation under VICP, a claim must be filed within 3 years after the first symptom of the vaccine injury. Death claims must be filed within 2 years of the vaccine-related death and not more than 4 years after the start of the first symptom of the vaccine-related injury from which the death occurred. When a new vaccine or a new injury/ condition is added to the Table, claims that do not meet the general filing guidelines must be filed within 2 years from the date the vaccine or injury/condition is added to the Table for injuries or deaths that occurred up to 8 years before the Table change (131). Persons of all ages who receive a VICP-covered vaccine might be eligible to file a claim. Additional information is available at https://www.hrsa.gov/vaccine-compensation/index. $\mathrm{html}$ or by calling 1-800-338-2382.

\section{Additional Resources}

\section{ACIP Statements}

- General Best Practice Guidelines for Immunization: Best Practices Guidance of the Advisory Committee on Immunization Practices (ACIP): https://www.cdc.gov/ vaccines/hcp/acip-recs/general-recs/index.html

- Immunization of Health Care Personnel: Recommendations of the Advisory Committee on Immunization Practices (ACIP), 2011. MMWR Recomm Rep 2011;60(No. RR-7): https:// www.cdc.gov/mmwr/preview/mmwrhtml/rr6007al.htm

- Recommended Adult Immunization Schedule for Ages 19 Years or Older, United States: https://www.cdc.gov/ vaccines/schedules/hcp/adult.html

- Recommended Child and Adolescent Immunization Schedule for Ages 18 Years or Younger, United States: https://www.cdc.gov/vaccines/schedules/hcp/imz/childadolescent.html 


\section{COVID-19 Vaccine Recommendations and Guidance}

- ACIP recommendations for the use of COVID-19 vaccines: https://www.cdc.gov/vaccines/hcp/acip-recs/ vacc-specific/covid-19.html

- Clinical Care Considerations for COVID-19 Vaccination (contains clinical guidance and links to current ACIP recommendations): https://www.cdc.gov/vaccines/covid19/clinical-considerations/index.html

- FDA COVID-19 Vaccines Page: https://www.fda.gov/ emergency-preparedness-and-response/coronavirusdisease-2019-covid-19/covid-19-vaccines

\section{Vaccine Information Sheets}

- IIV4 and RIV4: https://www.cdc.gov/vaccines/hcp/vis/ vis-statements/flu.pdf

- LAIV4: https://www.cdc.gov/vaccines/hcp/vis/visstatements/flulive.pdf

\section{Influenza Vaccine Package Inserts}

- https://www.fda.gov/vaccines-blood-biologics/vaccines/ influenza-virus-vaccine-quadrivalent-types-and-types-b

\section{CDC Influenza Antiviral Guidance}

- Influenza Antiviral Medications: Summary for Clinicians: https://www.cdc.gov/flu/professionals/antivirals/ summary-clinicians.htm

\section{Infectious Diseases Society of America Influenza Antiviral Guidance}

- Clinical Practice Guidelines by the Infectious Diseases Society of America: 2018 Update on Diagnosis, Treatment, Chemoprophylaxis, and Institutional Outbreak Management of Seasonal Influenza: https://academic.oup. com/cid/article/68/6/e1/5251935

\section{American Academy of Pediatrics Guidance}

- American Academy of Pediatrics Recommendations for Prevention and Control of Influenza in Children (Red Book Online): https://redbook.solutions.aap.org/selfserve/ sspage.aspx?selfservecontentid=influenza-resources

\section{Infectious Diseases Society of America Guidance for Vaccination of Immunocompromised Hosts}

- 2013 IDSA Clinical Practice Guideline for Vaccination of the Immunocompromised Host: https://academic.oup. $\mathrm{com} / \mathrm{cid} /$ article/58/3/e44/336537

\section{American College of Obstetricians and Gynecologists}

- Influenza Vaccination During Pregnancy, ACOG Committee Opinion No. 732: https://www.acog. org/clinical/clinical-guidance/committee-opinion/ articles/2018/04/influenza-vaccination-during-pregnancy

\section{References}

1. Barker WH. Excess pneumonia and influenza associated hospitalization during influenza epidemics in the United States, 1970-78. Am J Public Health 1986;76:761-5. PMID:3717461 https://doi.org/10.2105/ AJPH.76.7.761

2. Barker WH, Mullooly JP. Impact of epidemic type A influenza in a defined adult population. Am J Epidemiol 1980;112:798-811. PMID:7457471 https://doi.org/10.1093/oxfordjournals.aje.a113052

3. Poehling KA, Edwards KM, Griffin MR, et al. The burden of influenza in young children, 2004-2009. Pediatrics 2013;131:207-16. PMID:23296444 https://doi.org/10.1542/peds.2012-1255

4. Poehling KA, Edwards KM, Weinberg GA, et al.; New Vaccine Surveillance Network. The underrecognized burden of influenza in young children. N Engl J Med 2006;355:31-40. PMID:16822994 https://doi.org/10.1056/NEJMoa054869

5. Siston AM, Rasmussen SA, Honein MA, et al.; Pandemic H1N1 Influenza in Pregnancy Working Group. Pandemic 2009 influenza $\mathrm{A}(\mathrm{H} 1 \mathrm{~N} 1)$ virus illness among pregnant women in the United States. JAMA 2010;303:1517-25. PMID:20407061 https://doi.org/10.1001/ jama.2010.479

6. Mullooly JP, Bridges CB, Thompson WW, et al.; Vaccine Safety Datalink Adult Working Group. Influenza- and RSV-associated hospitalizations among adults. Vaccine 2007;25:846-55. PMID:17074423 https://doi. org/10.1016/j.vaccine.2006.09.041

7. Coleman BL, Fadel SA, Fitzpatrick T, Thomas SM. Risk factors for serious outcomes associated with influenza illness in high- versus lowand middle-income countries: Systematic literature review and metaanalysis. Influenza Other Respir Viruses 2018;12:22-9. PMID:29197154 https://doi.org/10.1111/irv.12504

8. Van Wormer JJ, King JP, Gajewski A, McLean HQ, Belongia EA. Influenza and workplace productivity loss in working adults. J Occup Environ Med 2017;59:1135-9. PMID:28759481 https://doi. org/10.1097/JOM.0000000000001120

9. Willis GA, Preen DB, Richmond PC, et al.; WAIVE Study Team. The impact of influenza infection on young children, their family and the health care system. Influenza Other Respir Viruses 2019;13:18-27. PMID:30137663 https://doi.org/10.1111/irv.12604

10. Fragaszy EB, Warren-Gash C, White PJ, et al.; Flu Watch Group. Effects of seasonal and pandemic influenza on health-related quality of life, work and school absence in England: results from the Flu Watch cohort study. Influenza Other Respir Viruses 2018;12:171-82. PMID:28991409 https://doi.org/10.1111/irv.12506

11. Fiore AE, Uyeki TM, Broder K, et al.. Prevention and control of influenza with vaccines: recommendations of the Advisory Committee on Immunization Practices (ACIP), 2010. MMWR Recomm Rep 2010;59(No. RR-8). PMID:20689501

12. CDC. How flu vaccine effectiveness and efficacy are measured: questions and answers. Atlanta, GA: US Department of Health and Human Services, CDC; 2016. https://www.cdc.gov/flu/professionals/ vaccination/effectivenessqa.htm

13. Rolfes MA, Foppa IM, Garg S, et al. Annual estimates of the burden of seasonal influenza in the United States: a tool for strengthening influenza surveillance and preparedness. Influenza Other Respir Viruses 2018;12:132-7. PMID:29446233 https://doi.org/10.1111/irv.12486 
14. Rolfes MA, Flannery B, Chung JR, et al.; US Influenza Vaccine Effectiveness (Flu VE) Network; Influenza Hospitalization Surveillance Network; Assessment Branch, Immunization Services Division, Centers for Disease Control and Prevention. Effects of influenza vaccination in the United States during the 2017-2018 influenza season. Clin Infect Dis 2019;69:1845-53. PMID:30715278 https://doi.org/10.1093/cid/ciz075

15. Patel A, Jernigan DB; 2019 nCoV CDC Response Team. Initial public health response and interim clinical guidance for the 2019 novel coronavirus outbreak-United States, December 31, 2019-February 4, 2020. MMWR Morb Mortal Wkly Rep 2020;69:140-6. PMID:32027631

16. World Health Organization. WHO Director-General's opening remarks at the media briefing on COVID-19; March 11, 2020. https://www.who. $\mathrm{int} /$ director-general/speeches/detail/who-director-general-s-openingremarks-at-the-media-briefing-on-covid-19---11-march-2020

17. CDC. COVID data tracker: United States COVID-19 cases and deaths by state. Atlanta, GA: US Department of Health and Human Services, CDC. https://covid.cdc.gov/covid-data-tracker/\#cases_casesper100klast7days

18. CDC. FluView. Weekly influenza surveillance report. Atlanta, GA: US Department of Health and Human Services, CDC. Accessed February 11, 2021. https://www.cdc.gov/flu/weekly/index.htm

19. Grohskopf LA, Alyanak E, Broder KR, et al. Prevention and control of seasonal influenza with vaccines: recommendations of the Advisory Committee on Immunization Practices-United States, 2020-21 influenza season. MMWR Recomm Rep 2020;69(No RR-8):1-24. PMID:32820746 https://doi.org/10.15585/mmwr.rr6908a1

20. Ahmed F. ACIP handbook for developing evidence-based recommendations, Version 1.2. Atlanta, GA: US Department of Health and Human Services, CDC; 2013.

21. Flucelvax Quadrivalent [Package Insert]. Holly Springs, NC: Seqirus; 2021.

22. Castilla J, Martínez-Baz I, Martínez-Artola V, et al.; Primary Health Care Sentinel Network; Network for Influenza Surveillance in Hospitals of Navarre. Decline in influenza vaccine effectiveness with time after vaccination, Navarre, Spain, season 2011/12. Euro Surveill 2013;18:5. PMID:23399423 https://doi.org/10.2807/ese.18.05.20388-en

23. Kissling E, Valenciano M, Larrauri A, et al. Low and decreasing vaccine effectiveness against influenza $\mathrm{A}(\mathrm{H} 3)$ in $2011 / 12$ among vaccination target groups in Europe: results from the I-MOVE multicentre casecontrol study. Euro Surveill 2013;18:5. PMID:23399425 https://doi. org/10.2807/ese.18.05.20390-en

24. Belongia EA, Sundaram ME, McClure DL, Meece JK, Ferdinands J, VanWormer JJ. Waning vaccine protection against influenza A (H3N2) illness in children and older adults during a single season. Vaccine 2015;33:246-51. PMID:24962752 https://doi.org/10.1016/j. vaccine.2014.06.052

25. Radin JM, Hawksworth AW, Myers CA, Ricketts MN, Hansen EA, Brice GT. Influenza vaccine effectiveness: Maintained protection throughout the duration of influenza seasons 2010-2011 through 2013-2014. Vaccine 2016;34:3907-12. PMID:27265447 https:/doi. org/10.1016/j.vaccine.2016.05.034

26. Ferdinands JM, Fry AM, Reynolds $S$, et al. Intraseason waning of influenza vaccine protection: Evidence from the US Influenza Vaccine Effectiveness Network, 2011-12 through 2014-15. Clin Infect Dis 2017;64:544-50. PMID:28039340

27. Puig-Barberà J, Mira-Iglesias A, Tortajada-Girbés M, et al.; Valencia Hospital Network for the Study of Influenza and other Respiratory Viruses (VAHNSI, Spain). Waning protection of influenza vaccination during four influenza seasons, 2011/2012 to 2014/2015. Vaccine 2017;35:5799-807. PMID:28941618 https://doi.org/10.1016/j. vaccine.2017.09.035

28. Ray GT, Lewis N, Klein NP, et al. Intraseason waning of influenza vaccine effectiveness. Clin Infect Dis 2019;68:1623-30. PMID:30204855 https://doi.org/10.1093/cid/ciy770
29. Kissling E, Nunes B, Robertson C, et al.; I-MOVE case-control study team. I-MOVE multicentre case-control study 2010/11 to 2014/15: is there within-season waning of influenza type/subtype vaccine effectiveness with increasing time since vaccination? Euro Surveill 2016;21:16. PMID:27124420 https://doi.org/10.2807/1560-7917. ES.2016.21.16.30201

30. Pebody R, Andrews N, McMenamin J, et al. Vaccine effectiveness of 2011/12 trivalent seasonal influenza vaccine in preventing laboratoryconfirmed influenza in primary care in the United Kingdom: evidence of waning intra-seasonal protection. Euro Surveill 2013;18:5. PMID:23399424 https://doi.org/10.2807/ese.18.05.20389-en

31. Ng Y, Nandar K, Chua LAV, et al. Evaluating the effectiveness of the influenza vaccine during respiratory outbreaks in Singapore's long term care facilities, 2017. Vaccine 2019;37:3925-31. PMID:31160102 https://doi.org/10.1016/j.vaccine.2019.03.054

32. Young BE, Mak TM, Ang LW, et al. Influenza vaccine failure in the tropics: a retrospective cohort study of waning effectiveness. Epidemiol Infect 2020;148:e299. PMID:33261680 https://doi.org/10.1017/ S0950268820002952

33. Powell LN, Bégué RE. Influenza vaccine effectiveness among children for the 2017-2018 season. J Pediatric Infect Dis Soc 2020;9:468-73. PMID:31774120 https://doi.org/10.1093/jpids/piz077

34. Mira-Iglesias A, López-Labrador FX, García-Rubio J, et al. Influenza vaccine effectiveness and waning effect in hospitalized older adults. Valencia Region, Spain, 2018/2019 season. Int J Environ Res Public Health 2021;18:1129. PMID:33514058 https://doi.org/10.3390/ ijerph18031129

35. Ferdinands JM, Gaglani M, Martin ET, et al. Waning vaccine effectiveness against influenza-associated hospitalizations among adults, 2015-2016 to 2018-2019, US Hospitalized Adult Influenza Vaccine Effectiveness Network. Clin Infect Dis 2021;2021:19. PMID:33462610

36. Madhi SA, Cutland CL, Kuwanda L, et al.; Maternal Flu Trial (Matflu) Team. Influenza vaccination of pregnant women and protection of their infants. N Engl J Med 2014;371:918-31. PMID:25184864 https://doi. org/10.1056/NEJMoa1401480

37. Tapia MD, Sow SO, Tamboura B, et al. Maternal immunisation with trivalent inactivated influenza vaccine for prevention of influenza in infants in Mali: a prospective, active-controlled, observer-blind, randomised phase 4 trial. Lancet Infect Dis 2016;16:1026-35. PMID:27261067 https://doi.org/10.1016/S1473-3099(16)30054-8

38. Steinhoff MC, Katz J, Englund JA, et al. Year-round influenza immunisation during pregnancy in Nepal: a phase 4, randomised, placebo-controlled trial. Lancet Infect Dis 2017;17:981-9. PMID:28522338 https://doi.org/10.1016/S1473-3099(17)30252-9

39. Eick AA, Uyeki TM, Klimov A, et al. Maternal influenza vaccination and effect on influenza virus infection in young infants. Arch Pediatr Adolesc Med 2011;165:104-11. PMID:20921345 https://doi. org/10.1001/archpediatrics.2010.192

40. Zaman K, Roy E, Arifeen SE, et al. Effectiveness of maternal influenza immunization in mothers and infants. N Engl J Med 2008;359:1555-64. PMID:18799552 https://doi.org/10.1056/NEJMoa0708630

41. CDC. The flu season. Atlanta, GA: US Department of Health and Human Services, CDC; 2015. https://www.cdc.gov/flu/about/season/ flu-season.htm

42. Young B, Sadarangani S, Jiang L, Wilder-Smith A, Chen MI. Duration of influenza vaccine effectiveness: A systematic review, meta-analysis, and meta-regression of test-negative design case-control studies. J Infect Dis 2018;217:731-41. PMID:29220496 https://doi.org/10.1093/infdis/jix632

43. Ferdinands JM, Alyanak E, Reed C, Fry AM. Waning of influenza vaccine protection: exploring the trade-offs of changes in vaccination timing among older adults. Clin Infect Dis 2020;70:1550-9. PMID:31257422 https://doi.org/10.1093/cid/ciz452

44. CDC. Immunization of health-care personnel: recommendations of the Advisory Committee on Immunization Practices (ACIP). MMWR Recomm Rep 2011;60(No. RR-7). PMID:22108587 
45. Pearson ML, Bridges CB, Harper SA. Advisory Committee on Immunization Practices (ACIP). Influenza vaccination of healthcare personnel: recommendations of the Healthcare Infection Control Practices Advisory Committee (HICPAC) and the Advisory Committee on Immunization Practices (ACIP). MMWR Recomm Rep 2006;55(No. RR-2). PMID:16498385

46. Kroger A, Bahta L, Hunter P. General best practice guidelines for immunization: best practices guidance of the Advisory Committee on Immunization Practices (ACIP). Atlanta, GA: US Department of Health and Human Services, CDC, Advisory Committee on Immunization Practices. https://www.cdc.gov/ vaccines/hcp/acip-recs/general-recs/index.html

47. Neuzil KM, Jackson LA, Nelson J, et al. Immunogenicity and reactogenicity of 1 versus 2 doses of trivalent inactivated influenza vaccine in vaccine-naive 5-8-year-old children. J Infect Dis 2006;194:1032-9. PMID:16991077 https://doi.org/10.1086/507309

48. Allison MA, Daley MF, Crane LA, et al. Influenza vaccine effectiveness in healthy 6- to 21-month-old children during the 2003-2004 season. J Pediatr 2006;149:755-62. PMID:17137887 https://doi.org/10.1016/j. jpeds.2006.06.036

49. Ritzwoller DP, Bridges CB, Shetterly S, Yamasaki K, Kolczak M, France EK. Effectiveness of the 2003-2004 influenza vaccine among children 6 months to 8 years of age, with 1 vs 2 doses. Pediatrics 2005;116:153-9. PMID:15995046 https://doi.org/10.1542/peds.2005-0049

50. Eisenberg KW, Szilagyi PG, Fairbrother G, et al.; New Vaccine Surveillance Network. Vaccine effectiveness against laboratory-confirmed influenza in children 6 to 59 months of age during the 2003-2004 and 2004-2005 influenza seasons. Pediatrics 2008;122:911-9. PMID:18977968 https://doi.org/10.1542/peds.2007-3304

51. ACOG Committee on Obstetric Practice. ACOG Committee opinion no. 732: influenza vaccination during pregnancy. Obstet Gynecol 2018;131:e109-14. PMID:29578985 https://doi.org/10.1097/ AOG.0000000000002588

52. ACOG Infectious Disease and Public Health Preparedness Expert Work Group. ACOG Committee opinion no. 741: maternal immunization. Obstet Gynecol 2018;131:e214-7. PMID:29794683 https://doi. org/10.1097/AOG.0000000000002662

53. Heikkinen T, Young J, van Beek E, et al. Safety of MF59-adjuvanted A/ H1N1 influenza vaccine in pregnancy: a comparative cohort study. Am J Obstet Gynecol 2012;207:177.e1-8 PMID:22939717. PMID:22939717 https://doi.org/10.1016/j.ajog.2012.07.007

54. Oppermann M, Fritzsche J, Weber-Schoendorfer C, et al. A(H1N1) v2009: a controlled observational prospective cohort study on vaccine safety in pregnancy. Vaccine 2012;30:4445-52. PMID:22564554 https://doi.org/10.1016/j.vaccine.2012.04.081

55. Pasternak B, Svanström H, Mølgaard-Nielsen D, et al. Vaccination against pandemic A/H1N1 2009 influenza in pregnancy and risk of fetal death: cohort study in Denmark. BMJ 2012;344:e2794. PMID:22551713 https://doi.org/10.1136/bmj.e2794

56. Sammon CJ, Snowball J, McGrogan A, de Vries CS. Evaluating the hazard of foetal death following $\mathrm{H} 1 \mathrm{~N} 1$ influenza vaccination; a population based cohort study in the UK GPRD. PLoS One 2012;7:e51734. PMID:23341865 https://doi.org/10.1371/journal. pone. 0051734

57. Chambers CD, Johnson D, Xu R, et al.; OTIS Collaborative Research Group. Risks and safety of pandemic H1N1 influenza vaccine in pregnancy: birth defects, spontaneous abortion, preterm delivery, and small for gestational age infants. Vaccine 2013;31:5026-32. PMID:24016809 https://doi.org/10.1016/j.vaccine.2013.08.097

58. Irving SA, Kieke BA, Donahue JG, et al.; Vaccine Safety Datalink. Trivalent inactivated influenza vaccine and spontaneous abortion. Obstet Gynecol 2013;121:159-65. PMID:23262941 https://doi.org/10.1097/ AOG.0b013e318279f56f
59. Huang WT, Tang FW, Yang SE, Chih YC, Chuang JH. Safety of inactivated monovalent pandemic (H1N1) 2009 vaccination during pregnancy: a population-based study in Taiwan. Vaccine 2014;32:6463-8. PMID:25285884 https://doi.org/10.1016/j.vaccine.2014.09.054

60. Ma F, Zhang L, Jiang R, et al. Prospective cohort study of the safety of an influenza $\mathrm{A}(\mathrm{H} 1 \mathrm{~N} 1)$ vaccine in pregnant Chinese women. Clin Vaccine Immunol 2014;21:1282-7. PMID:24990911 https://doi.org/10.1128/ CVI.00375-14

61. Chambers CD, Johnson DL, Xu R, et al.; OTIS Collaborative Research Group. Safety of the 2010-11, 2011-12, 2012-13, and 2013-14 seasonal influenza vaccines in pregnancy: birth defects, spontaneous abortion, preterm delivery, and small for gestational age infants, a study from the cohort arm of VAMPSS. Vaccine 2016;34:4443-9. PMID:27449682 https://doi.org/10.1016/j.vaccine.2016.06.054

62. McMillan M, Porritt K, Kralik D, Costi L, Marshall H. Influenza vaccination during pregnancy: a systematic review of fetal death, spontaneous abortion, and congenital malformation safety outcomes. Vaccine 2015;33:2108-17. PMID:25758932 https://doi.org/10.1016/j. vaccine.2015.02.068

63. Bratton KN, Wardle MT, Orenstein WA, Omer SB. Maternal influenza immunization and birth outcomes of stillbirth and spontaneous abortion: a systematic review and meta-analysis. Clin Infect Dis 2015;60:e11-9. PMID:25409473 https://doi.org/10.1093/cid/ciu915

64. Donahue JG, Kieke BA, King JP, et al. Association of spontaneous abortion with receipt of inactivated influenza vaccine containing H1N1pdm09 in 2010-11 and 2011-12. Vaccine 2017;35:5314-22. PMID:28917295 https://doi.org/10.1016/j.vaccine.2017.06.069

65. Donahue JG, Kieke BA, King JP, et al. Inactivated influenza vaccine and spontaneous abortion in the Vaccine Safety Datalink in 2012-13, 2013-14, and 2014-15. Vaccine 2019;37:6673-81. PMID:31540812 https://doi.org/10.1016/j.vaccine.2019.09.035

66. DiazGranados CA, Dunning AJ, Kimmel M, et al. Efficacy of high-dose versus standard-dose influenza vaccine in older adults. N Engl J Med 2014;371:635-45. PMID:25119609 https://doi.org/10.1056/ NEJMoa1315727

67. Dunkle LM, Izikson R, Patriarca P, et al.; PSC12 Study Team. Efficacy of recombinant influenza vaccine in adults 50 years of age or older. N Engl J Med 2017;376:2427-36. PMID:28636855 https://doi. org/10.1056/NEJMoa1608862

68. Van Buynder PG, Konrad S, Van Buynder JL, et al. The comparative effectiveness of adjuvanted and unadjuvanted trivalent inactivated influenza vaccine (TIV) in the elderly. Vaccine 2013;31:6122-8. PMID:23933368 https://doi.org/10.1016/j.vaccine.2013.07.059

69. Falsey AR, Treanor JJ, Tornieporth N, Capellan J, Gorse GJ. Randomized, double-blind controlled phase 3 trial comparing the immunogenicity of high-dose and standard-dose influenza vaccine in adults 65 years of age and older. J Infect Dis 2009;200:172-80. PMID:19508159 https:// doi.org/10.1086/599790

70. Frey SE, Reyes MR, Reynales $\mathrm{H}$, et al. Comparison of the safety and immunogenicity of an MF59 -adjuvanted with a non-adjuvanted seasonal influenza vaccine in elderly subjects. Vaccine 2014;32:5027-34. PMID:25045825 https://doi.org/10.1016/j.vaccine.2014.07.013

71. Food and Drug Administration. Clinical review: Fluzone High-Dose. Silver Spring, MD: US Department of Health and Human Services, Food and Drug Administration; 2014. http://wayback.archive-it. org/7993/20170723030559/https://www.fda.gov/downloads/ BiologicsBloodVaccines/Vaccines/ApprovedProducts/UCM422372.pdf

72. Young-Xu Y, Van Aalst R, Mahmud SM, et al. Relative vaccine effectiveness of high-dose versus standard-dose influenza vaccines among Veterans Health Administration patients. J Infect Dis 2018;217:1718-27. PMID:29452380 https://doi.org/10.1093/infdis/jiy088 
73. Shay DK, Chillarige Y, Kelman J, et al. Comparative effectiveness of high-dose versus standard-dose influenza vaccines among US Medicare beneficiaries in preventing postinfluenza deaths during 2012-2013 and 2013-2014. J Infect Dis 2017;215:510-7. PMID:28329311 https:// doi.org/10.1093/infdis/jiw641

74. Izurieta HS, Thadani N, Shay DK, et al. Comparative effectiveness of high-dose versus standard-dose influenza vaccines in US residents aged 65 years and older from 2012 to 2013 using Medicare data: a retrospective cohort analysis. Lancet Infect Dis 2015;15:293-300. PMID:25672568 https://doi.org/10.1016/S1473-3099(14)71087-4

75. Richardson DM, Medvedeva EL, Roberts CB, Linkin DR; Centers for Disease Control and Prevention Epicenter Program. Comparative effectiveness of high-dose versus standard-dose influenza vaccination in community-dwelling veterans. Clin Infect Dis 2015;61:171-6. PMID:25829001 https://doi.org/10.1093/cid/civ261

76. Gravenstein S, Davidson HE, Taljaard M, et al. Comparative effectiveness of high-dose versus standard-dose influenza vaccination on numbers of US nursing home residents admitted to hospital: a cluster-randomised trial. Lancet Respir Med 2017;5:738-46. PMID:28736045 https://doi. org/10.1016/S2213-2600(17)30235-7

77. Lee JKH, Lam GKL, Shin T, et al. Efficacy and effectiveness of high-dose versus standard-dose influenza vaccination for older adults: a systematic review and meta-analysis. Expert Rev Vaccines 2018;17:435-43. PMID:29715054 https://doi.org/10.1080/14760584.2018.1471989

78. Fluzone High-Dose Quadrivalent [Package Insert]. Swiftwater, PA: Sanofi Pasteur; 2020.

79. Chang LJ, Meng Y, Janosczyk H, Landolfi V, Talbot HK; QHD00013 Study Group. Safety and immunogenicity of high-dose quadrivalent influenza vaccine in adults $\geq 65$ years of age: A phase 3 randomized clinical trial. Vaccine 2019;37:5825-34 https://doi.org/10.1016/j. vaccine.2019.08.016. PMID:31431411

80. Flublok Quadrivalent [Package Insert]. Meriden, CT: Protein Sciences; 2021.

81. De Serres G. Selection bias in the comparison between adjuvanted and unadjuvanted influenza vaccine effectiveness. Vaccine 2014;32:6596. PMID:24380683 https://doi.org/10.1016/j.vaccine.2013.12.032

82. Mannino S, Villa M, Apolone G, et al. Effectiveness of adjuvanted influenza vaccination in elderly subjects in northern Italy. Am J Epidemiol 2012;176:527-33. PMID:22940713 https://doi. org/10.1093/aje/kws313

83. Lapi F, Marconi E, Simonetti M, et al. Adjuvanted versus nonadjuvanted influenza vaccines and risk of hospitalizations for pneumonia and cerebro/cardiovascular events in the elderly. Expert Rev Vaccines 2019;18:663-70. PMID:31155968 https://doi.org/10.1080/1476058 4.2019.1622418

84. Fluad Quadrivalent [Package Insert]. Holly Springs, NC: Seqirus; 2020.

85. Beran J, Reynales H, Poder A, et al. Prevention of influenza during mismatched seasons in older adults with an MF59-adjuvanted quadrivalent influenza vaccine: a randomised, controlled, multicentre, phase 3 efficacy study. Lancet Infect Dis 2021;21:1027-37 https://doi. org/10.1016/S1473-3099(20)30694-0. PMID:33577767

86. Essink B, Fierro C, Rosen J, et al. Immunogenicity and safety of MF59adjuvanted quadrivalent influenza vaccine versus standard and alternate B strain MF59-adjuvanted trivalent influenza vaccines in older adults. Vaccine 2020;38:242-50 https://doi.org/10.1016/j.vaccine.2019.10.021. PMID:31635976

87. Food and Drug Administration. Clinical review: Fluad Quadrivalent. Silver Spring, MD: US Department of Health and Human Services, Food and Drug Administration; 2020.

88. Izurieta HS, Chillarige Y, Kelman J, et al. Relative effectiveness of cellcultured and egg-based influenza vaccines among elderly persons in the United States, 2017-2018. J Infect Dis 2019;220:1255-64. PMID:30561688 https://doi.org/10.1093/infdis/jiy716
89. Izurieta HS, Chillarige Y, Kelman J, et al. Relative effectiveness of influenza vaccines among the United States elderly, 2018-2019. J Infect Dis 2020;222:278-87. PMID:32100009 https://doi.org/10.1093/ infdis/jiaa080

90. Izurieta HS, Lu M, Kelman J, et al. Comparative effectiveness of influenza vaccines among U.S. Medicare beneficiaries ages 65 years and older during the 2019-20 season. Clin Infect Dis 2020;ciaa1727. PMID:33211809 https://doi.org/10.1093/cid/ciaa1727

91. Schmader KE, Liu CK, Harrington T, et al. Safety, reactogenicity, and health-related quality of life after trivalent adjuvanted vs trivalent highdose inactivated influenza vaccines in older adults: a randomized clinical trial. JAMA Netw Open 2021;4:e2031266. PMID:33443580 https:// doi.org/10.1001/jamanetworkopen.2020.31266

92. Rubin LG, Levin MJ, Ljungman P, et al.; Infectious Diseases Society of America. 2013 IDSA clinical practice guideline for vaccination of the immunocompromised host. Clin Infect Dis 2014;58:e44-100. PMID:24311479 https://doi.org/10.1093/cid/cit684

93. CDC. Influenza antiviral medications: summary for clinicians. Atlanta, GA: US Department of Health and Human Services, CDC; 2021. https:/www.cdc.gov/flu/professionals/antivirals/summary-clinicians.htm

94. Woo EJ. Allergic reactions after egg-free recombinant influenza vaccine: reports to the US Vaccine Adverse Event Reporting System. Clin Infect Dis 2015;60:777-80. PMID:25428412 https://doi.org/10.1093/cid/ciu948

95. Woo EJ, Moro PL, Cano M, Jankosky C. Postmarketing safety surveillance of trivalent recombinant influenza vaccine: reports to the Vaccine Adverse Event Reporting System. Vaccine 2017;35:5618-21. PMID:28886946 https://doi.org/10.1016/j.vaccine.2017.08.047

96. Woo EJ, Moro PL. Postmarketing safety surveillance of quadrivalent recombinant influenza vaccine: Reports to the vaccine adverse event reporting system. Vaccine 2021;39:1812-7. PMID:33678452 https:// doi.org/10.1016/j.vaccine.2021.02.052

97. Uyeki TM, Zane SB, Bodnar UR, et al.; Alaska/Yukon Territory Respiratory Outbreak Investigation Team. Large summertime influenza A outbreak among tourists in Alaska and the Yukon Territory. Clin Infect Dis 2003;36:1095-102. PMID:12715302 https://doi. org/10.1086/374053

98. Mutsch M, Tavernini M, Marx A, et al. Influenza virus infection in travelers to tropical and subtropical countries. Clin Infect Dis 2005;40:1282-7. PMID:15825030 https://doi.org/10.1086/429243

99. Ratnam I, Black J, Leder K, et al. Incidence and risk factors for acute respiratory illnesses and influenza virus infections in Australian travellers to Asia. J Clin Virol 2013;57:54-8. PMID:23380660 https://doi. org/10.1016/j.jcv.2013.01.008

100. Millman AJ, Kornylo Duong K, Lafond K, Green NM, Lippold SA, Jhung MA. Influenza outbreaks among passengers and crew on two cruise ships: a recent account of preparedness and response to an everpresent challenge. J Travel Med 2015;22:306-11. PMID:26031322 https://doi.org/10.1111/jtm.12215

101. FluMist Quadrivalent [Package Insert]. Gaithersburg, MD: MedImmune; 2021.

102. Rapivab (peramivir for injection) [Package Insert]. Durham, NC: BioCryst; 2017.

103. Xofluza (baloxavir marboxil) [Package Insert]. San Francisco, CA: Genentech; 2018. https:/www.accessdata.fda.gov/drugsatfda_docs/ label/2018/210854s000lbl.pdf

104. Food and Drug Administration. Guidance for industry: bioavailability and bioequivalence studies for orally administered drug products: general considerations. Silver Spring, MD: US Department of Health and Human Services, Food and Drug Administration; 2003. 
105. Kerzner B, Murray AV, Cheng E, et al. Safety and immunogenicity profile of the concomitant administration of ZOSTAVAX and inactivated influenza vaccine in adults aged 50 and older. J Am Geriatr Soc 2007;55:1499-507. PMID:17908055 https://doi. org/10.1111/j.1532-5415.2007.01397.x

106. Levin MJ, Buchwald UK, Gardner J, et al. Immunogenicity and safety of zoster vaccine live administered with quadrivalent influenza virus vaccine. Vaccine 2018;36:179-85. PMID:28830693 https://doi. org/10.1016/j.vaccine.2017.08.029

107. Frenck RW Jr, Gurtman A, Rubino J, et al. Randomized, controlled trial of a 13-valent pneumococcal conjugate vaccine administered concomitantly with an influenza vaccine in healthy adults. Clin Vaccine Immunol 2012;19:1296-303. PMID:22739693 https://doi. org/10.1128/CVI.00176-12

108. Schwarz TF, Flamaing J, Rümke HC, et al. A randomized, double-blind trial to evaluate immunogenicity and safety of 13 -valent pneumococcal conjugate vaccine given concomitantly with trivalent influenza vaccine in adults aged $\geq 65$ years. Vaccine 2011;29:5195-202. PMID:21619909 https://doi.org/10.1016/j.vaccine.2011.05.031

109. McNeil SA, Noya F, Dionne M, et al. Comparison of the safety and immunogenicity of concomitant and sequential administration of an adult formulation tetanus and diphtheria toxoids adsorbed combined with acellular pertussis (Tdap) vaccine and trivalent inactivated influenza vaccine in adults. Vaccine 2007;25:3464-74. PMID:17270320 https://doi.org/10.1016/j.vaccine.2006.12.047

110. Nakashima K, Aoshima M, Ohfuji S, et al. Immunogenicity of simultaneous versus sequential administration of a 23 -valent pneumococcal polysaccharide vaccine and a quadrivalent influenza vaccine in older individuals: a randomized, open-label, non-inferiority trial. Hum Vaccin Immunother 2018;14:1923-30. PMID:29561248 https://doi.org/10.1080/21645515.2018.1455476

111. Song JY, Cheong HJ, Tsai TF, et al. Immunogenicity and safety of concomitant MF59-adjuvanted influenza vaccine and 23-valent pneumococcal polysaccharide vaccine administration in older adults. Vaccine 2015;33:4647-52. PMID:25980426 https://doi.org/10.1016/j. vaccine.2015.05.003

112. Sukumaran L, McCarthy NL, Kharbanda EO, et al. Safety of tetanus toxoid, reduced diphtheria toxoid, and acellular pertussis and influenza vaccinations in pregnancy. Obstet Gynecol 2015;126:1069-74. PMID:26444109 https://doi.org/10.1097/AOG.0000000000001066

113. Stockwell MS, Broder K, LaRussa P, et al. Risk of fever after pediatric trivalent inactivated influenza vaccine and 13-valent pneumococcal conjugate vaccine. JAMA Pediatr 2014;168:211-9. PMID:24395025 https://doi.org/10.1001/jamapediatrics.2013.4469

114. Walter EB, Klein NP, Wodi AP, et al. Fever after influenza, diphtheriatetanus-acellular pertussis, and pneumococcal vaccinations. Pediatrics 2020;145:e20191909. PMID:32029684 https://doi.org/10.1542/ peds.2019-1909

115. Duffy J, Weintraub E, Hambidge SJ, et al.; Vaccine Safety Datalink. Febrile seizure risk after vaccination in children 6 to 23 months. Pediatrics 2016;138:e20160320. PMID:27273711 https://doi. org/10.1542/peds.2016-0320
116. Li R, Stewart B, McNeil MM, et al. Post licensure surveillance of influenza vaccines in the Vaccine Safety Datalink in the 2013-2014 and 2014-2015 seasons. Pharmacoepidemiol Drug Saf 2016;25:928-34. PMID:27037540 https://doi.org/10.1002/pds.3996

117. Patterson JL, Carapetian SA, Hageman JR, Kelley KR. Febrile seizures. Pediatr Ann 2013;42:249-54. PMID:24295158 https://doi. org/10.3928/00904481-20131122-09

118. Nolan T, Bernstein DI, Block SL, et al.; LAIV Study Group. Safety and immunogenicity of concurrent administration of live attenuated influenza vaccine with measles-mumps-rubella and varicella vaccines to infants 12 to 15 months of age. Pediatrics 2008;121:508-16 https:// doi.org/10.1542/peds.2007-1064. PMID:18310199

119. Lum LC, Borja-Tabora CF, Breiman RF, et al. Influenza vaccine concurrently administered with a combination measles, mumps, and rubella vaccine to young children. Vaccine 2010;28:1566-74. PMID:20003918 https://doi.org/10.1016/j.vaccine.2009.11.054

120. Shingrix [Package Insert]. Research Triangle Park, NC: GlaxoSmithKline; 2017.

121. Fluad Quadrivalent [Package Insert]. Holly Springs, NC: Seqirus; 2021.

122. Heplisav-B [Package Insert]. Emeryville, CA: Dynavax; 2017.

123. Schwarz TF, Aggarwal N, Moeckesch B, et al. Immunogenicity and safety of an adjuvanted herpes zoster subunit vaccine coadministered with seasonal influenza vaccine in adults aged 50 years or older. J Infect Dis 2017;216:1352-61. PMID:29029224 https://doi.org/10.1093/ infdis/jix481

124. Afluria Quadrivalent [Package Insert]. Parkville, Victoria, Australia: Seqirus; 2021.

125. Fluarix Quadrivalent [Package Insert]. Dresden, Germany: GlaxoSmithKline; 2021.

126. FluLaval Quadrivalent [Package Insert]. Quebec City, QC, Canada: ID Biomedical Corporation of Quebec; 2021.

127. Fluzone Quadrivalent [Package Insert]. Swiftwater, PA: Sanofi Pasteur; 2021.

128. Fluzone High-Dose Quadrivalent [Package Insert]. Swiftwater, PA: Sanofi Pasteur; 2021.

129. Food and Drug Administration. Clinical review: Flucelvax Quadrivalent. Silver Spring, MD: US Department of Health and Human Services, Food and Drug Administration; 2016.

130. Food and Drug Administration. Clinical review: Flucelvax Quadrivalent. Silver Spring, MD: US Department of Health and Human Services, Food and Drug Administration; 2021.

131. Health Resources and Services Administration. What you need to know about the National Vaccine Injury Compensation Program (VICP). Washington, DC: US Department of Health and Human Services, Health Resources and Services Administration; 2019. https://www. hrsa.gov/sites/default/files/hrsa/vaccine-compensation/resources/aboutvaccine-injury-compensation-program-booklet.pdf 


\section{Disclosure of Relationship and Unlabeled Use}

All authors have completed and submitted the International Committee of Medical Journal Editors form for the disclosure of potential conflicts of interest. Jill M. Ferdinands reports travel-related support that is unrelated to this work from the Institute for Influenza Epidemiology, which is funded in part by Sanofi Pasteur. Helen Keipp Talbot reports serving as the secretary for the National Foundation for Infectious Diseases, for which she receives no payment. No other potential conflicts of interest were disclosed. This report includes discussion of the unlabeled use of influenza vaccines in the instance of influenza vaccination of persons with a history of egg allergy. A history of severe allergic reaction (e.g., anaphylaxis) to the vaccine or any of its components (which include egg for some vaccines) is a labeled contraindication to receipt of most IIV4s and LAIV4. However, ACIP recommends that persons with a history of allergic reaction of any severity to egg should receive any licensed, recommended influenza vaccine that is appropriate for their age and health status. Persons with a history of severe allergic reaction to egg who receive egg-based vaccines (i.e., vaccines other than cell culture-based inactivated influenza vaccine [ccIIV4] or recombinant influenza vaccine [RIV4]) should be vaccinated in an inpatient or outpatient medical setting (including, but not necessarily limited to, hospitals, clinics, health departments, and physician offices); vaccine administration in such instances should be supervised by a health care provider who is able to recognize and manage severe allergic reactions. No postvaccination waiting period is recommended specifically for egg-allergic persons. However, ACIP recommends that vaccine providers consider observing patients seated or supine for 15 minutes following administration of any vaccine (regardless of allergy history) to decrease the risk for injury should syncope occur. 


\section{Advisory Committee on Immunization Practices (ACIP), July 1, 2020-June 30, 2021}

Chair: José R. Romero, MD, University of Arkansas for Medical Sciences, Little Rock, Arkansas.

Executive Secretary: Amanda Cohn, MD, National Center for Immunization and Respiratory Diseases, CDC Atlanta, Georgia.

Members: Kevin A. Ault, MD, University of Kansas Medical Center, Kansas City, Kansas; Lynn Bahta, MPH, Minnesota Department of Health, St. Paul, Minnesota; Beth P. Bell, MD, University of Washington, Seattle, Washington; Henry Bernstein, DO, Zucker School of Medicine at Hofstra/Northwell Cohen Children's Medical Center, New Hyde Park, New York; Wilbur H. Chen, MD, University of Maryland School of Medicine, Baltimore, Maryland; Matthew F. Daley, MD, Kaiser Permanente Colorado, Aurora, Colorado; Sharon E. Frey, MD, Saint Louis University Medical School, St. Louis, Missouri; Camille Nelson Kotton, MD, Harvard Medical School, Boston, Massachusetts; Grace M. Lee, MD, Lucile Packard Children's Hospital, Stanford University School of Medicine, Stanford, California; Sarah S. Long, MD, Drexel University College of Medicine, Philadelphia, Pennsylvania; Veronica V. McNally, JD, Franny Strong Foundation, West Bloomfield, Michigan; Katherine A. Poehling, MD, Wake Forest School of Medicine, Winston-Salem, North Carolina; Pablo J. Sánchez, MD, The Research Institute at Nationwide Children's Hospital, Columbus, Ohio; Helen Keipp Talbot, MD, Vanderbilt University, Nashville, Tennessee.

Ex Officio Members: Centers for Medicare and Medicaid Services, Mary Beth Hance, Baltimore, Maryland; Food and Drug Administration, Doran Fink, MD, PhD, Silver Spring, Maryland; Health Resources and Services Administration, Mary Rubin, MD, Rockville, Maryland; Indian Health Service, Thomas Weiser, MD, Portland, Oregon; Office of Infectious Disease and HIV/AIDS Policy, David Kim, MD, Washington, DC; National Institutes of Health, John Beigel, MD, Bethesda, Maryland.

Liaison Representatives: American Academy of Family Physicians, Pamela G. Rockwell, DO, Ann Arbor, Michigan; American Academy of Pediatrics, Committee on Infectious Diseases, Yvonne Maldonado, MD, Stanford, California; American Academy of Pediatrics, Red Book Editor, David Kimberlin, MD, Birmingham, Alabama; American Academy of Physician Assistants, Marie-Michèle Léger, MPH, PA-C, Alexandria, Virginia; American College Health Association, Thevy Chai, MD, Chapel Hill, North Carolina; American College Health Association, (alternate) Sharon McMullen, MPH, Ithaca, New York; American College of Nurse Midwives, Carol E. Hayes, MN, MPH, Atlanta, Georgia; American College of Nurse Midwives (alternate), Pamela M. Meharry, PhD, Chicago, Illinois; American College of Obstetricians and Gynecologists, Linda O'Neal Eckert, MD, Seattle, Washington; American College of Physicians, Jason M. Goldman, MD, Boca Raton, Florida; American Geriatrics Society, Kenneth Schmader, MD, Durham, North Carolina; America's Health Insurance Plans, Robert Gluckman, MD, Beaverton, Oregon; American Immunization Registry Association, Rebecca Coyle, MSEd, Washington, DC; American Medical Association, Sandra Adamson Fryhofer, MD, Atlanta, Georgia; American Nurses Association, Charles (Chad) Rittle, DNP, Pittsburgh, Pennsylvania; American Osteopathic Association, Stanley E. Grogg, DO, Tulsa, Oklahoma; American Pharmacists Association, Stephan L. Foster, PharmD, Memphis, Tennessee; Association of Immunization Managers, Molly Howell, MPH, Bismarck, North Dakota; Association for Prevention Teaching and Research, W. Paul McKinney, MD, Louisville, Kentucky; Association of State and Territorial Health Officials, Nirav Shah, MD, JD, Little Rock, Arkansas; Biotechnology Industry Organization, Phyllis A. Arthur, MBA, Washington, DC; Council of State and Territorial Epidemiologists, Christine Hahn, MD, Boise, Idaho; Council of State and Territorial Epidemiologists, (alternate) Susan Lett, MD, Boston, Massachusetts; Canadian National Advisory Committee on Immunization, Caroline Quach, MD, Montreal, Québec, Canada; Infectious Diseases Society of America, Carol J. Baker, MD, Houston, Texas; International Society for Travel Medicine, Elizabeth D. Barnett, MD, Boston, Massachusetts; National Association of County and City Health Officials, Matthew Zahn, MD, Santa Ana, California; National Association of County and City Health Officials, (alternate) Jeffrey Duchin, MD, Seattle, Washington; National Association of Pediatric Nurse Practitioners, Patricia A. Stinchfield, MS, St. Paul, Minnesota; National Foundation for Infectious Diseases, William Schaffner, MD, Nashville, Tennessee; National Foundation for Infectious Diseases, (alternate) Marla Dalton, Bethesda, Maryland; National Medical Association, Patricia Whitley-Williams, MD, New Brunswick, New Jersey; Pediatric Infectious Diseases Society, Sean O'Leary, MD, Aurora, Colorado; Pediatric Infectious Diseases Society, (alternate) Mark H. Sawyer, MD, San Diego, California; Pharmaceutical Research and Manufacturers of America, Corey Robertson, MD, Swiftwater, Pennsylvania; Society for Adolescent Health and Medicine, Amy B. Middleman, MD, Oklahoma City, Oklahoma; Society for Healthcare Epidemiology of America, Marci Drees, MD, Philadelphia, Pennyslvania.

\section{ACIP Influenza Vaccine Work Group}

Chair: H. Keipp Talbot, MD, Nashville, Tennessee.

Members: Robert Atmar, MD, Houston, Texas; Kevin Ault, MD, Kansas City, Missouri; Edward Belongia, MD, Marshfield, Wisconsin; Henry Bernstein, DO, Hempstead, New York; Sarah Coles, MD, Phoenix, Arizona; Michael Cooper, PhD, Bethesda, Maryland; Clarence Creech, MD, Nashville, Tennessee; Jeff Duchin, MD, Seattle, Washington; Sandra Adamson Fryhofer, MD, Atlanta, Georgia; Robyn Harrison, MD, Edmonton, Alberta, Canada; Denise Jamieson, MD, Atlanta, Georgia; Wendy Keitel, MD, Houston, Texas; Camille Kotton, MD, Boston, Massachusetts; Marie-Michèle Léger, MPH, Alexandria, Virginia; Susan Lett, MD, Boston, Massachusetts; Jamie Loehr, MD, Ithaca, New York; Mark Mulligan, MD, New York, New York; Flor M. Munoz, MD, Houston, Texas; Kathleen M. Neuzil, MD, Baltimore, Maryland; Cynthia Nolletti, MD, Silver Spring, Maryland; William Schaffner, MD, Nashville, Tennessee; Robert Schechter, MD, Richmond, California; Kenneth Schmader, MD, Durham, North Carolina; Tamara Sheffield, MD, Salt Lake City, Utah; Angela Sinilaite, MPH, Ottawa, Ontario, Canada; Patricia Stinchfield, MS, St. Paul, Minnesota; Peter Szilagyi, MD, Los Angeles, California; Susan Wollersheim, MD, Silver Spring, Maryland; Emmanuel (Chip) Walter, MD, Durham, North Carolina; Matthew Zahn, MD, Santa Ana, California. 

The Morbidity and Mortality Weekly Report (MMWR) Series is prepared by the Centers for Disease Control and Prevention (CDC) and is available free

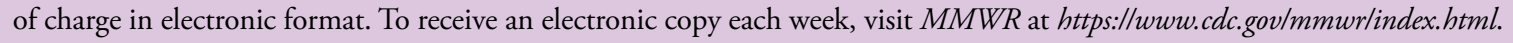

Readers who have difficulty accessing this PDF file may access the HTML file at https://www.cdc.gov/mmwr/volumes/70/rr/rr7005a1.htm?s_ cid=rr7005a1_w. Address all inquiries about the MMWR Series to Editor-in-Chief, MMWR Series, Mailstop V25-5, CDC, 1600 Clifton Rd., N.E., Atlanta, GA 30329-4027 or to mmwrq@cdc.gov.

All material in the MMWR Series is in the public domain and may be used and reprinted without permission; citation as to source, however, is appreciated. MMWR and Morbidity and Mortality Weekly Report are service marks of the U.S. Department of Health and Human Services.

Use of trade names and commercial sources is for identification only and does not imply endorsement by the U.S. Department of Health and Human Services.

References to non-CDC sites on the Internet are provided as a service to $M M W R$ readers and do not constitute or imply endorsement of these organizations or their programs by CDC or the U.S. Department of Health and Human Services. CDC is not responsible for the content of these sites. URL addresses listed in $M M W R$ were current as of the date of publication.

ISSN: 1057-5987 (Print) 